\title{
Linear Frequency Domain and Harmonic Balance Predictions of Dynamic Derivatives
}

\author{
A. Da Ronch, ${ }^{*}$ M. Ghoreyshi, ${ }^{\dagger}$ K.J. Badcock, ${ }^{\ddagger}$ \\ Department of Engineering, University of Liverpool, Liverpool, UK L69 3BX, United Kingdom \\ S. Görtz, ${ }^{\S}$ M. Widhalm, ${ }^{\circledR}$ R. P. Dwight, ॥ \\ Institute of Aerodynamics and Flow Technology, German Aerospace Center (DLR), 38108 Braunschweig, Germany \\ and M.S. Campobasso ** \\ School of Engineering, University of Glasgow, Glasgow, Scotland G12 8QQ, United Kingdom
}

\begin{abstract}
Dynamic derivatives are used to represent the influence of the aircraft rates on the aerodynamic forces and moments needed for flight dynamics studies. These values have traditionally been estimated by processing measurements made from periodic forced motions applied to wind tunnel models. The use of Computational Fluid Dynamics has potential to supplement this approach. This paper considers the problem of the fast computation of forced periodic motions using the Euler equations. Three methods are evaluated. The first is computation in the time domain, and this provides the benchmark solution in the sense that the time accurate solution is obtained. Two acceleration techniques in the frequency domain are compared. The first uses an harmonic solution of the linearised problem (referred to as the linear frequency domain approach). The second uses the Harmonic Balance method, which approximates the nonlinear problem using a number of Fourier modes. These approaches are compared in the sense of their ability to predict dynamic derivatives and their computational cost. The standard NACA aerofoil CT cases, the SDM fighter model geometry and the DLR F12 passenger jet wind tunnel model are used as test cases. Compared to time accurate simulations an orderof-magnitude reduction in CPU costs is achieved for flows with a narrow frequency spectrum and moderate amplitudes, as the solution does not evolve through transients to reach periodicity.
\end{abstract}

\section{Introduction}

In general, aerodynamic models of forces and moments are non-linear functions of the aircraft states. Fortunately, for flight simulation the model introduced by Bryan $^{1}$ is often applicable. This model uses a linear relationship between the forces and moments, and the flight states, with the coefficients referred to as aerodynamic derivatives. There are three types of derivative, namely static, dynamic and control. ${ }^{2}$ Dynamic derivatives are calculated from observing the response of aerodynamic forces and moments to translational and rotational motions. Dynamic derivatives influence the aerodynamic damping of aircraft motions and are used to evaluate the longitudinal short period, lateral pure roll and lateral Dutch roll properties of the aircraft. ${ }^{3}$

Aerodynamic forces and moments can be estimated via analytical, semi-empirical, computational fluid dynamics or experimental methods. A combination of data sheets, linear aerodynamic theory, and empirical relations have met with success due to their simplicity, but their generality is limited. The US Air Force DATCOM, ${ }^{4}$ for example, has received widespread use. DATCOM produces aerodynamic derivatives based on the aircraft geometry and flight conditions, and is based on a database obtained for conventional aircraft configurations, restricting validity. Aircraft designers use semi-empirical methods such as DATCOM for estimating the static and dynamic derivatives during conceptual design, while wind tunnel testing is traditionally applied later in the design cycle.

\footnotetext{
*Ph.D. Student, corresponding author. Tel.: +44(0)151-7948035, Email: A.Da-Ronch@liverpool.ac.uk

$\dagger$ Research Assistant

¥Professor, SMAIAA

$\S$ Researcher Engineer

T Researcher Engineer

॥ Aerospace Engineer

** Lecturer
} 
Wind tunnel testing has traditionally been used to produce derivatives for production aircraft based on scale models. This can be a time consuming and expensive process. The physical realism of wind tunnel data is well known, but can be limited by blockage, scaling, and Reynolds-number effects together with support interference issues that prevent the proper modelling of the full-scale vehicle behaviour.

A relatively new tool for evaluating dynamic derivatives is Computational Fluid Dynamics (CFD). Navier-Stokes CFD solvers have reached a level of robustness and maturity to support routine use on relatively inexpensive computer clusters. The computation of static stability derivatives can be done with off-the-shelf CFD tools. The prediction of dynamic derivatives requires the ability to compute the aerodynamic response to time-dependent prescribed motions which are used to excite the aerodynamics of interest. CFD has potential for complementing experimental testing techniques for obtaining these aerodynamic parameters. The physical limitations and kinematic restrictions of wind tunnel testing including model motion as well as the interference effects of the model support are not factors in the computational analysis. Physical effects can be separated from the CFD solutions in a way which can be difficult from wind tunnel or flight test data. However, for predicting dynamic derivatives, significant computer resources are required since unsteady simulations are needed.

Since many of the traditional wind-tunnel testing techniques rely on harmonic forced-oscillation tests, it would be helpful to have a computational tool that takes advantage of the periodic nature of the motion to decrease the computational costs incurred by unsteady simulations. The computational methods presented in this paper, the Harmonic Balance (HB) and the Linear Frequency Domain (LFD) method provide the ability to efficiently approximate the aerodynamics resulting from small, periodic and unsteady perturbations of the geometry of an aircraft configuration. There is however the question of the influence of the approximations on the derivative predictions. The evaluation of the computational benefits and the predictive limitations is the subject of this paper.

The paper begins with a description of the time domain, LFD and HB methods. Results are presented then to compare the dynamic derivative predictions obtained by the LFD, HB and time domain methods.

\section{Numerical Approach}

The Harmonic Balance (HB) and Linearised Frequency Domain (LFD) methods compared in the current work are implemented in different CFD codes. The approach taken is to benchmark each against the underlying unsteady flow solver. In the current section the underlying flow solvers are first summarised, and then the HB and LFD methods described.

\section{A. Time Domain Formulations}

\section{University of Liverpool $(P M B)$}

The main features of the Parallel Multiblock (PMB) solver are described in Badcock et al. ${ }^{5}$ A fully implicit steady solution of the Reynolds Averaged Navier Stokes (RANS) or Euler equations is obtained by advancing the solution forward in time by solving the discrete nonlinear system of equations

$$
\frac{\mathbf{W}^{n+1}-\mathbf{W}^{n}}{\Delta t}=-\mathbf{R}\left(\mathbf{W}^{n+1}\right)
$$

The term on the right hand side, called the residual, is the discretisation of the convective terms, given here by Osher's approximate Riemann solver, ${ }^{6}$ MUSCL interpolation ${ }^{7}$ and Van Albada's limiter. Eq. (1) is a nonlinear system of algebraic equations which is solved by an implicit method, ${ }^{5}$ the main features of which are an approximate linearisation to reduce the size and condition number of the linear system, and the use of a preconditioned Krylov subspace method to calculate the updates.

The steady state solver is applied to unsteady problems within a pseudo time stepping iteration ${ }^{8}$ which at each real time step is written as

$$
\left[\left(\frac{1}{\Delta t}-\frac{3}{2 \Delta t^{*}}\right) \mathbf{I}+\frac{\partial \mathbf{R}}{\partial \mathbf{W}}\right] \Delta \mathbf{W}=-\left(\mathbf{R}(\mathbf{W})+\frac{3 \mathbf{W}^{n+1}-4 \mathbf{W}^{n}+\mathbf{W}^{n-1}}{2 \Delta t^{*}}\right)
$$

where $\Delta t^{*}$ is the real time step. Periodicity can be used to approximate the initial solution for the pseudo time stepping at each real time step. At each iteration a file is written to the local disk with the converged solution at that real time step. On the next cycle this file is read to provide the initial solution for the pseudo time stepping, and on convergence to the next real time solution, the original file is overwritten with the updated solution. As the solution approaches a 
periodic state the pseudo time stepping converges quickly because it starts from an excellent initial guess. In this way results can be obtained from time marching in a very efficient manner.

\section{German Aerospace Center (TAU)}

The DLR TAU code ${ }^{9,10}$ (TAU) is not one code but a modern massively parallel software system for the simulation of flows around complex geometries from low subsonic to hypersonic flow regimes. The different modules of TAU can be used stand alone or in a more efficient way within a Python scripting framework which allows for intermodule communication without file I/O by using common memory allocations. The unsteady compressible ReynoldsAveraged Navier-Stokes (RANS) flow solver is based on hybrid unstructured grids with a finite volume discretization. The flow solver uses an edge-based dual-cell approach, either cell-vertex or cell-centred, employing either a secondorder central scheme or a variety of upwind schemes with linear reconstruction for second order accuracy.

As for the PMB solver, unsteady simulations uses Jameson's dual-time-stepping method ${ }^{8}$ to integrate the timedependent equations in the time-domain. Additionally, the solver respects the geometric conservation law both grid deformation as well as bodies in arbitrary motion can be simulated. For the pseudo time stepping various explicit Runge-Kutta and a semi-implicit LUSGS (Lower-Upper Symmetric Gauss-Seidel) scheme are available by enhancing convergence acceleration with a geometrical multi-grid algorithm and local time-stepping.

Apart from that, the Tau code includes an adjoint-solver for gradient based numerical shape optimization. The discrete adjoint method ${ }^{11}$ consists of the explicit construction of the exact flux Jacobians of the spatial discretization with respect to the unknown flow variables allowing the adjoint equations to be formulated and solved and is a main part of many different linear solvers, i.e. linear frequency domain solver or error estimation methods.

\section{University of Glasgow (COSA)}

The structured Navier-Stokes solver COSA code is a multigrid finite volume cell-centered code. It solves the integral conservation laws in generalized curvilinear coordinates making use of a second order discretisation method. The discretisation of the convective fluxes is based on Van Leer's MUSCL extrapolations and the approximate Riemann solver of Roe's flux-difference splitting. The discretisation of the viscous fluxes uses centred finite-differences. The set of nonlinear algebraic equations resulting from the space-discretisation of the conservation laws is solved with an explicit approach based on the use of a four-stage Runge-Kutta smoother. The convergence rate is greatly enhanced by means of local time-stepping, variable-coefficient central implicit residual smoothing and a full-approximation storage multigrid algorithm. When solving problems at very low flow speed computational accuracy and high levels of convergence speed are maintained by using a carefully designed low-speed preconditioning. ${ }^{12}$

In the case of unsteady problems, Jameson's dual-time-stepping method ${ }^{8}$ is used to integrate the time-dependent equations in the time-domain. The interested reader is referred to references ${ }^{12-14}$ for further details on the COSA solver and a thorough validation of its inviscid and viscous capabilities for steady and unsteady problems.

\section{B. The Harmonic Balance Method}

As an alternative to time marching, the Harmonic Balance method ${ }^{15}$ allows for a direct calculation of the periodic state. Write the semi-discrete form as a system of ordinary differential equations

$$
\mathbf{I}(t)=\frac{d \mathbf{W}(t)}{d t}+\mathbf{R}(t)=0
$$

Consider the solution $\mathbf{W}$ and residual $\mathbf{R}$ to be periodic in time and a function of $\omega$,

$$
\begin{aligned}
& \mathbf{W}(t) \approx \hat{\mathbf{W}}_{0}+\sum_{n=1}^{N_{H}}\left(\hat{\mathbf{W}}_{a_{n}} \cos (\omega n t)+\hat{\mathbf{W}}_{b_{n}} \sin (\omega n t)\right) \\
& \mathbf{R}(t) \approx \hat{\mathbf{R}}_{0}+\sum_{n=1}^{N_{H}}\left(\hat{\mathbf{R}}_{a_{n}} \cos (\omega n t)+\hat{\mathbf{R}}_{b_{n}} \sin (\omega n t)\right)
\end{aligned}
$$

giving a system of $N_{T}=2 N_{H}+1$ equations in $N_{T}$ unknown harmonic terms and can be expressed as

$$
\omega \mathbf{A} \hat{\mathbf{W}}+\hat{\mathbf{R}}=\mathbf{0}
$$


where $\mathbf{A}$ is a $N_{T} \times N_{T}$ matrix containing the entries $\mathbf{A}\left(n+1, N_{H}+n+1\right)=n$ and $\mathbf{A}\left(N_{H}+n+1, n+1\right)=-n$, and $\hat{\mathbf{W}}$ and $\hat{\mathbf{R}}$ are vectors of the Fourier coefficients.

The difficulty with solving Eq. (6) is in finding a relationship between $\hat{\mathbf{R}}$ and $\hat{\mathbf{W}}$. To avoid this problem the system is converted back to the time domain. The solution is split into $N_{T}$ discrete equally spaced sub intervals over the period $T=2 \pi / \omega$

$$
\mathbf{W}_{h b}=\left(\begin{array}{c}
\mathbf{W}\left(t_{0}+\Delta t\right) \\
\mathbf{W}\left(t_{0}+2 \Delta t\right) \\
\vdots \\
\mathbf{W}\left(t_{0}+T\right)
\end{array}\right) \quad \mathbf{R}_{h b}=\left(\begin{array}{c}
\mathbf{R}\left(t_{0}+\Delta t\right) \\
\mathbf{R}\left(t_{0}+2 \Delta t\right) \\
\vdots \\
\mathbf{R}\left(t_{0}+T\right)
\end{array}\right)
$$

where $\Delta t=2 \pi /\left(N_{T} \omega\right)$. Then there is a transformation matrix ${ }^{16}$ which allows Eq. (6) to be written as

$$
\omega \mathbf{D} \mathbf{W}_{h b}+\mathbf{R}_{h b}=0
$$

where the components of $\mathbf{D}$ are defined by

$$
D_{i, j}=\frac{2}{N_{T}} \sum_{k=1}^{N_{H}} k \sin \left(2 \pi k(j-i) / N_{T}\right)
$$

One can then apply pseudo time marching to the Harmonic Balance equation

$$
\frac{d \mathbf{W}_{h b}}{d t}+\omega D \mathbf{W}_{h b}+\mathbf{R}_{h b}=0
$$

This equation is solved using an implicit method ${ }^{17}$ similar to the one used for the underlying flow solver PMB.

The HB method has also been implemented using the explicit multigrid Navier-Stokes solver COSA. The only difference between the PMB and COSA implementation of the HB method is that the latter solves the harmonic balance system of Eq. (8) by means of the aforementioned explicit multigrid method.

\section{Linearized Frequency Domain Method}

The Linearized Frequency Domain (LFD) method ${ }^{18,19}$ is obtained by linearizing Eq. (6), in which the residual $\hat{\mathbf{R}}$ is considered as a function of the grid point locations, $\mathbf{x}$, the grid point velocities, $\dot{\mathbf{x}}$, and flow solution, $\mathbf{W}$. Assuming an unsteady motion with a small amplitude, the unsteady terms can be expressed as superposition of a steady mean state and a perturbation, which is expressed by a Fourier series

$$
\begin{aligned}
\mathbf{W}(t) & \approx \hat{\mathbf{W}}_{0}+\tilde{\mathbf{W}}(t), \quad\|\tilde{\mathbf{W}}\| \ll\left\|\hat{\mathbf{W}}_{0}\right\| \\
\mathbf{x}(t) & \approx \hat{\mathbf{x}}_{0}+\tilde{\mathbf{x}}(t), \quad\|\tilde{\mathbf{x}}\| \ll\left\|\hat{\mathbf{x}}_{0}\right\| \\
\dot{\mathbf{x}}(t) & \approx \dot{\tilde{\mathbf{x}}}(t) .
\end{aligned}
$$

When linearizing about the steady mean state, Eq. (6) results in the following complex valued linear system of equations for the $n^{\text {th }}$ mode index

$$
\left(\begin{array}{cc}
\partial \mathbf{R} / \partial \mathbf{W} & \omega n \mathbf{I} \\
-\omega n \mathbf{I} & \partial \mathbf{R} / \partial \mathbf{W}
\end{array}\right)\left\{\begin{array}{c}
\hat{\mathbf{W}}_{a_{n}} \\
\hat{\mathbf{W}}_{b_{n}}
\end{array}\right\}=-\left(\begin{array}{cc}
\partial \mathbf{R} / \partial \mathbf{x} & \omega n \partial \mathbf{R} / \partial \dot{\mathbf{x}} \\
-\omega n \partial \mathbf{R} / \partial \dot{\mathbf{x}} & \partial \mathbf{R} / \partial \mathbf{x}
\end{array}\right)\left\{\begin{array}{c}
\hat{\mathbf{X}}_{a_{n}} \\
\hat{\mathbf{X}}_{b_{n}}
\end{array}\right\} .
$$

Derivatives of the residual are all evaluated at the steady mean state $\left(\hat{\mathbf{W}}_{0}, \hat{\mathbf{x}}_{0}\right)$, here dropped for convenience. This system of equations can be written in the form of a linear equation, $\mathbf{A} \mathbf{x}=\mathbf{b}$. The accuracy of the result will depend on the degree to which the dual assumptions of small perturbations and linearity are satisfied.

The Jacobian $\partial \mathbf{R} / \partial \mathbf{W}$ has been obtained previously in the context of the discrete adjoint method by analytic differentiating the flow solver. Considerable attention has been given to ensure that the evaluation of the Jacobian and matrix-vector products involving the Jacobian are efficient in terms of memory and time and requires no more than four times the memory requirements of the non-linear code. The frequency domain residual however requires two products of a vector with the Jacobian, and hence a single evaluation is approximately $20 \%-60 \%$ more expensive than a non-linear residual on the same case. 
The terms $\partial \mathbf{R} / \partial \mathbf{x}$ and $\partial \mathbf{R} / \partial \dot{\mathbf{x}}$, which represent the predescribed small periodic deformation of the grid, are evaluated using central finite differences

$$
\begin{aligned}
\frac{\partial \mathbf{R}}{\partial \mathbf{x}} \approx \frac{\mathbf{R}\left(\hat{\mathbf{W}}_{0}, \hat{\mathbf{x}}_{0}+\epsilon \tilde{\mathbf{x}}, 0\right)-\mathbf{R}\left(\hat{\mathbf{W}}_{0}, \hat{\mathbf{x}}_{0}-\epsilon \tilde{\mathbf{x}}, 0\right)}{2 \epsilon} & \frac{\partial \mathbf{R}\left(\hat{\mathbf{W}}_{0}, \hat{\mathbf{x}}_{0}, \epsilon \dot{\tilde{\mathbf{x}}}\right)-\mathbf{R}\left(\hat{\mathbf{W}}_{0}, \hat{\mathbf{x}}_{0},-\epsilon \dot{\tilde{\mathbf{x}}}\right)}{2 \epsilon}
\end{aligned}
$$

where $\epsilon$ is a small number chosen to minimize the error. It might be small enough to avoid non-linear effects in the result and large enough so that the numerical noise is negligible. A good practice to get accurate results is the experimentation with various values.

\section{Method of Data Analysis}

The prediction of dynamic derivatives based on the time-domain methods and the LFD solver was performed in the frequency domain using a cubic Lagrange polynomial approximation of the Fourier integral. More details on the implemented scheme are found in Da Ronch et al. ${ }^{20}$ The unstructured solvers output the mean value and the first harmonic of the flow solution without additional postprocessing. A postprocessing utility was implemented for the extraction of the zeroth and first harmonic flow solution computed from the time-domain solution.

A different approach was adopted for the HB solver, whose solution is computed at $N_{T}=2 N_{H}+1$ equally spaced points in time over one cycle. In other words,

$$
\mathbf{W}(x, y, z, t) \approx \hat{\mathbf{W}}_{0}(x, y, z)+\sum_{n=1}^{N_{H}}\left(\hat{\mathbf{W}}_{a_{n}}(x, y, z) \cos (\omega n t)+\hat{\mathbf{W}}_{b_{n}}(x, y, z) \sin (\omega n t)\right)
$$

where $\hat{\mathbf{W}}_{0}, \hat{\mathbf{W}}_{a_{n}}$ and $\hat{\mathbf{W}}_{b_{n}}$ are the Fourier coefficients of a flow variable, $\mathbf{W}(x, y, z, t)$. This expression is easily re-written in matrix form as

$$
\underbrace{\left\{\begin{array}{c}
\mathbf{W}_{1} \\
\mathbf{W}_{2} \\
\vdots \\
\mathbf{W}_{N_{T}}
\end{array}\right\}}_{\mathbf{W}^{*}}=\underbrace{\left[\begin{array}{cccccc}
1 & \cos \left(\omega t_{1}\right) & \sin \left(\omega t_{1}\right) & \ldots & \cos \left(N_{H} \omega t_{1}\right) & \sin \left(N_{H} \omega t_{1}\right) \\
1 & \cos \left(\omega t_{2}\right) & \sin \left(\omega t_{2}\right) & \ldots & \cos \left(N_{H} \omega t_{2}\right) & \sin \left(N_{H} \omega t_{2}\right) \\
\vdots & \vdots & \vdots & \ddots & \vdots & \vdots \\
1 & \cos \left(\omega t_{N_{T}}\right) & \sin \left(\omega t_{N_{T}}\right) & \ldots & \cos \left(N_{H} \omega t_{N_{T}}\right) & \sin \left(N_{H} \omega t_{N_{T}}\right)
\end{array}\right]}_{\mathbf{E}^{-1}} \underbrace{\left[\begin{array}{c}
\hat{\mathbf{W}}_{0} \\
\hat{\mathbf{W}}_{a_{1}} \\
\hat{\mathbf{W}}_{b_{1}} \\
\vdots \\
\hat{\mathbf{W}}_{a_{N_{H}}} \\
\hat{\mathbf{W}}_{b_{N_{H}}}
\end{array}\right\}}_{\tilde{\mathbf{W}}}
$$

where $\mathbf{W}^{*}$ is the vector of the flow variable at $2 N_{H}+1$ equally spaced points in time over one period and $\mathbf{E}^{-1}$ is the matrix that is the inverse discrete Fourier transform operator. The time instances at which the HB solution is known are denoted by $t_{i}=t_{0}+i \Delta t, i=1,2, \ldots, N_{T}$. The Fourier coefficients of the flow variable are computed as

$$
\tilde{\mathbf{W}}=\mathbf{E} \mathbf{W}^{*}
$$

Dynamic derivatives, as well as the real and imaginary parts of the flow variable, are determined directly from the Fourier coefficients without any additional transformation in the time domain.

The issue whether the assumptions of the HB and the LFD methods impact the prediction of dynamic derivatives is addressed for a test case that will be presented to some extent in the following sections. The AGARD CT5 case is selected for the pitching NACA 0012 airfoil. A fully-unsteady calculation was computed using the PMB and TAU solvers and the pitching moment loops are shown in Fig. 1(a) and 1(b), respectively. The frequency spectrum was extracted from the time signals of the moment coefficient. The mean value (zeroth harmonic) and the fundamental harmonic were retained to reconstruct in the time-domain the signal indicated by Time Domain - $1^{\text {st }}$ Har in the figure. The dynamic derivatives of the moment coefficient are obtained from the real and imaginary parts of the first harmonic component. The signal includes the component of time-average over the simulated cycles and the background motion, neglecting terms of higher order. The 1 mode HB and the LFD solvers were also run for the same test case and the corresponding moment coefficient is shown against the respective time-domain results. The ellipse identified by 
the HB solution has nearly the same shape of that of the 1 harmonic time-domain signal. However, the 1 mode solution fails to predict the location of the shock wave with accuracy, as shown in Fig. 2, and the mean value results slightly underpredicted. The dynamic derivatives predicted for this case are in close agreement with the time-domain values for the first mode HB solution. The LFD result for the pitching moment coefficient shows a phase shift in counterclockwise direction which is a-priori not clear what influence caused that shift.

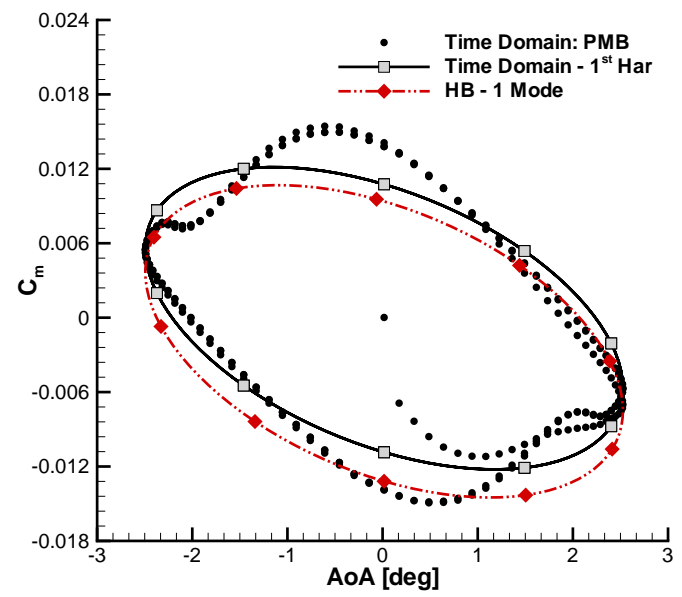

(a) PMB and $\mathrm{HB}$ results

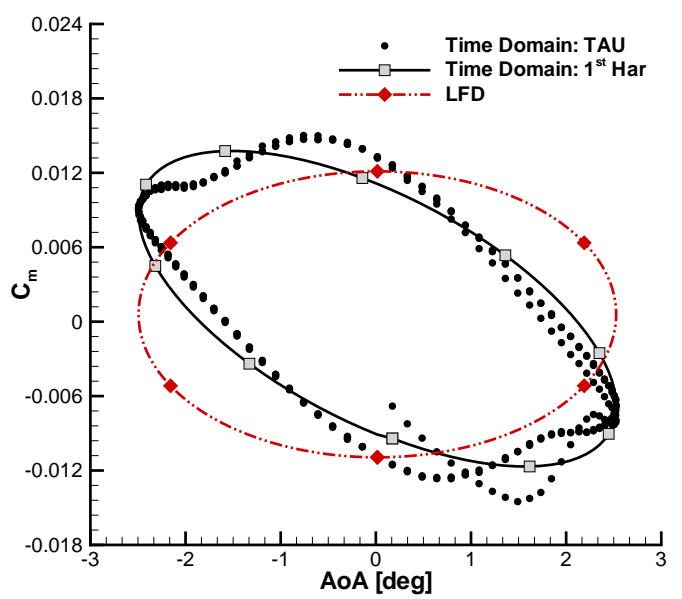

(b) TAU and LFD results

Figure 1. Comparison of the first harmonic pitching moment coefficient for the time-domain, HB and LFD solutions for NACA 0012; the test case is the AGARD CT5

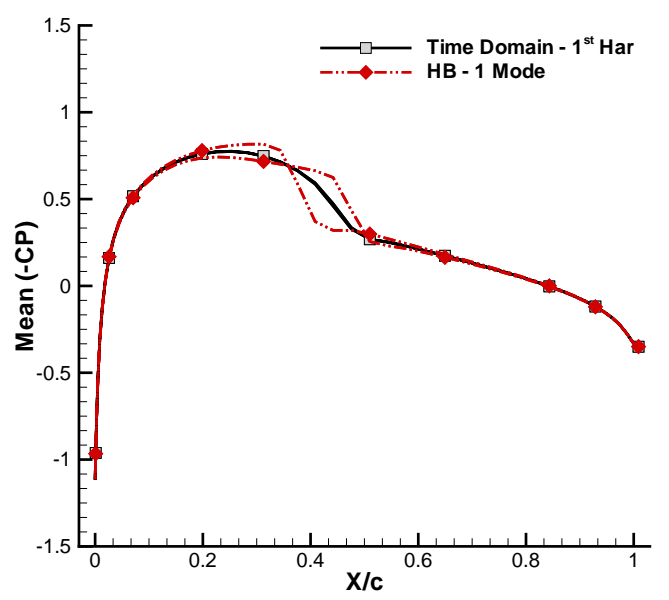

Figure 2. Mean value of pressure coefficient distribution for the PMB and the 1 Mode HB solutions for NACA 0012; the test case is the AGARD CT5

Significantly different results were obtained for the AGARD CT5 case using the LFD and the 1 mode HB solution. Our understanding is that the step at which the harmonic truncation is done impacts the solution. The LFD method consists of the linearization of the equations governing the flow with respect to a small perturbation superimposed over a base flow. The base state is the steady solution for the LFD method. It takes into account reference states, like a shock at a define location, but cannot model dynamic motion of shock waves as observed for the AGARD CT5 case. For the HB method, the truncation to a finite number of harmonics is done in the resolution of the equations and 
the base state is a time-average solution over the periodic motion. As will be demonstrated, non-linear phenomena featuring shock wave motion and vortical flow developments can be handled by the HB method.

\section{Results and Discussion}

\section{A. NACA 0012 Airfoil}

Experimental data for the unsteady aerodynamics of the NACA 0012 airfoil undergoing oscillatory pitch motions are available. ${ }^{21,22}$ Measured quantities include the pressure at 30 locations distributed on the airfoil surface. These data were collected at several time intervals. No transition tripping was applied in the experiments, and corrections corresponding to a steady interference have been applied to the measured quantities. There were some questions about unsteady interference effects on the experimental data. However, the deviation between numerical and experimental data is not the emphasis of the present work which is instead on the quality of the LFD and HB results compared to the time domain predictions.

Two challenging cases were selected among the available flow configurations in the AGARD data set for the NACA 0012 geometry pitching at one-quarter of the chord. The flow conditions related to the AGARD CT2 and CT5 were retained to provide a comparison for numerical results. The test cases summarized in Table 1 are characterized by high pitching amplitudes and low frequencies. The flow fields for the two cases are characterized by the formation of a strong and highly dynamic shock wave experiencing Tijdeman and Seebass's ${ }^{23}$ type-B shock motion. However, for case CT2 the steady state solution does not feature a shock wave. It is observed that the magnitude of the periodic change in shock strength is larger than the mean steady shock strength and, thereby, the shock wave disappears during a part of its background motion. For case CT5, the steady solution includes a virtually symmetric shock wave which periodically appears and disappears on the upper and lower surfaces as consequence of the harmonic motion.

The HB and the LFD methods were compared with the time-domain PMB, COSA and TAU solutions and the available experimental measurements. To investigate the influence of the number of modes on the accuracy of the HB solution, calculations were performed using up to 7 Fourier modes.

\begin{tabular}{lll}
\hline \hline AGARD & CT2 & CT5 \\
\hline Mach number, $M$ & 0.6 & 0.755 \\
Mean incidence, $\alpha_{0}$ & $3.16^{\circ}$ & $0.016^{\circ}$ \\
Pitch amplitude, $\alpha_{A}$ & $4.59^{\circ}$ & $2.51^{\circ}$ \\
Reduced frequency, $k$ & 0.0811 & 0.0814 \\
\hline \hline
\end{tabular}

Table 1. Description of the NACA 0012 test cases

The block structured grids and the unstructured grid of the NACA 0012 airfoil are shown in Fig. 3. The twodimensional domain extends 15 chords to the farfield for the three-block structured grid. The C-type mesh has 97 nodes on the airfoil and 33 points in the normal direction. The wake behind the airfoil is discretised using 17 points in the streamwise direction. The total number of points is 8646. An unstructured mesh was generated for use with TAU. The farfield is circular and located at 100 chords from the airfoil surface. The unstructured grid consists of 2280 points, 4300 prisms in the domain and 130 quadrilaterals at the wall. The structured grid for the COSA solver consists of 7425 points, 33 points in the normal direction and 180 nodes on the airfoil. The farfield is located at 20 chords from the solid wall.

The periodic motion of the aerofoil is defined by the angle of attack as a function of time by

$$
\alpha(t)=\alpha_{0}+\alpha_{\mathrm{A}} \sin (\omega t)
$$

where $\alpha_{0}$ is the mean incidence, $\alpha_{\mathrm{A}}$ the amplitude of pitching oscillation, and $\omega$ the angular frequency of the motion which is related to the reduced frequency $k$ by

$$
k=\omega c /\left(2 U_{\infty}\right)
$$

The airfoil chord and the free-stream speed are denoted, respectively, by $c$ and $U_{\infty}$. 


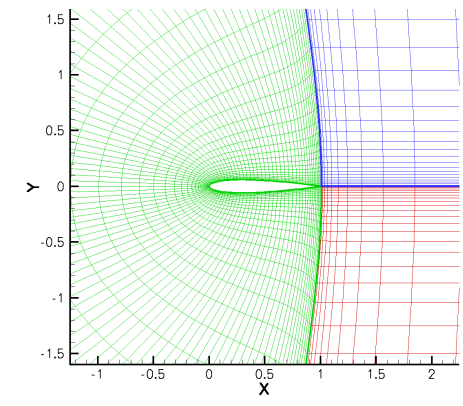

(a) Three-block structured grid for the PMB and HB solvers

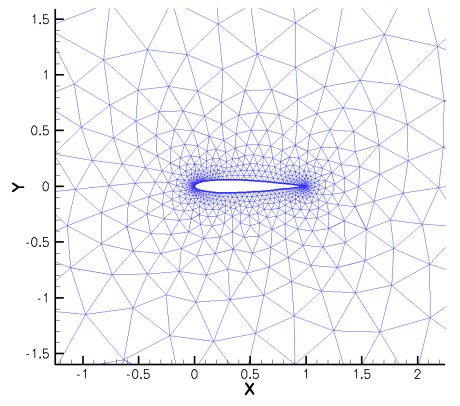

(b) Unstructured grid for the TAU and LFD solvers

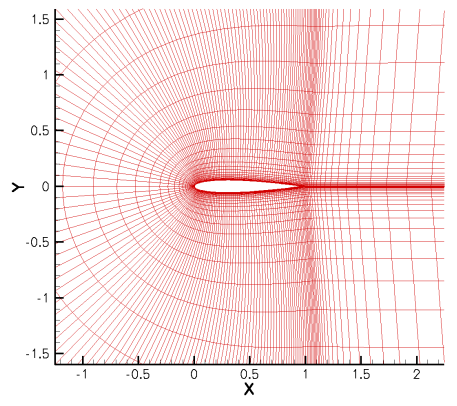

(c) Structured grid for the time-domain and $\mathrm{HB}$ COSA solvers

Figure 3. View of the grids for the NACA 0012

\section{Numerical Setup}

The primary objective of the work is to assess the accuracy of the HB and LFD solutions compared to the respective time-domain methods. However, the computational efficiency in terms of CPU time is also addressed to establish if the acceleration techniques represent feasible alternatives to the time-accurate solver with a favourable time reduction. To this purpose, the choice of the numerical parameters is clearly important. The main numerical parameters used in the test cases are summarized in Table 2.

The choice of the numerical parameters for all solvers led to well converged solutions for all test cases. Timedomain analyses were simulated for 3 oscillatory cycles. For the PMB and TAU solvers, 100 real time steps per period were considered. For the time-domain COSA analyses, 128 time intervals per period were used. Preliminary calculations using the time-domain PMB solver were run for time steps per cycle up to 1024. The increasing number of time steps did not noticeably influence the predictions for all test cases and virtually identical results were obtained. A reduction of the L 2 norm of the residual by 8 orders of magnitude was the stopping criterion for the PMB and TAU analyses, whilst for the COSA analyses the multigrid integration was stopped when the maximum root-mean-square of the residuals of the four equations was smaller than $10^{-10}$.

For the implicit HB solver, it was found that the use of a local adaptive CFL number in pseudo-marching the $\mathrm{HB}$ equations had a favourable outcome on the overall run time. The increase in CPU time for large CFL numbers was caused by the degradation of the preconditioner as the time step was increased. To avoid this negative issue, the CFL number was limited up to a maximum value. The maximum CFL number was progressively reduced as more harmonics were retained in the solution because of the increased time for the evaluation of each pseudo iteration.

All time-domain and HB COSA analyses were carried out using the multigrid solver with 3 grid levels, and performing 10 smoothed Runge-Kutta cycles on the coarsest level. On the finest and medium levels, 5 smoothed Runge-Kutta cycles were instead performed. In all cases, the CFL number was held constant from the beginning to the end of the calculation. The initial conditions of the time-domain and HB COSA analyses were the same as those used for the corresponding PMB analyses.

The L2 norm of the residual for the HB, LFD and HB COSA solvers are shown in Fig. 4 for all test cases. Convergence to the prescribed tolerance required more iterations for the AGARD CT5, due to a more complex flow physics. The steady state solutions are also included for reference. Nearly the same number of pseudo-iterations in the HB solver were required to reach convergence for all analyses, except for the 7 modes, for the first test case. For the second test case, the number of iterations increased for increasing number of retained Fourier modes. For the HB COSA results, the number of multi-grid iterations did not increase with the number of harmonics and, in particular, the poorest performance was obtained for a different number of modes in the two test cases. A final remark is that the performance of the LFD method did not change noticeably.

\section{Test Case 1: AGARD CT2}

The flow field of the AGARD CT2 case is characterised by the formation of a strong and highly dynamic shock wave experiencing Tijdeman and Seebass's ${ }^{23}$ type-B shock motion. The magnitude of the periodic change in shock strength is larger than the mean steady shock strength and, thereby, the shock wave disappears during a part of its background 


\begin{tabular}{lllllll}
\hline \hline AGARD & CT2 & CT5 & & & & \\
\hline Time Domain: $P M B$ & & & & & & \\
Number of cycles & 3 & 3 & & & & \\
Number of time-steps per cycle & 100 & 100 & & & & \\
CFL number & 500 & 500 & & & & \\
\hline$H B$ & $(1-7)$ & $(1,2)$ & $(3)$ & $(4)$ & $(5)$ & $(6,7)$ \\
Max number of pseudo-iterations & 300 & 300 & 300 & 300 & 300 & 300 \\
Min CFL number & 50 & 50 & 30 & 25 & 20 & 10 \\
Max CFL number & 500 & 500 & 300 & 250 & 200 & 150 \\
\hline \hline Time Domain: TAU & & & & & & \\
Number of cycles & 3 & 3 & & & & \\
Number of time-steps per cycle & 100 & 100 & & & & \\
CFL number & 20 & 20 & & & & \\
Relaxation solver & LU-SGS & LU-SGS & & & & \\
\hline LFD & & & & & & \\
CFL number & 20 & 20 & & & & \\
Relaxation solver & LU-SGS & LU-SGS & & & & \\
Modal amplitude factor & $10^{-4}$ & $10^{-4}$ & & & & \\
\hline \hline Time Domain: COSA & & & & & & \\
Number of cycles & 3 & 3 & & & & \\
Number of time-steps per cycle & 128 & 128 & & & \\
CFL number & 3 & 2 & & & \\
\hline HB-COSA & 3 & 1 & & & \\
CFL number & & & & & & \\
\hline \hline
\end{tabular}

Table 2. Numerical parameters for the NACA 0012 test cases; the values in parenthesis indicate the number of harmonics retained in the HB solution

motion. The normal force and the pitching moment coefficients referred to body axes are considered for the comparison of the HB and the LFD methods with the time-accurate solutions. The loops of the integrated aerodynamic coefficients against the instantaneous angle of attack are shown in Fig. 5. The HB solution is included for 1, 2, 3 and 7 modes. The convergence of the time-domain solution to a steady harmonic output was achieved within the first cycle of motion. As can be seen from the lift force coefficient, Fig. 5(a), for the time-accurate simulation, non-linear effects are present for the dynamic derivatives already and which appear in the pitching moment coefficient, Fig. 5(b), as harmonics of more than second order. The solutions computed using 2, 3 and 7 modes agree well in predicting the force loop. However, the solutions retaining only the first harmonic miss some of the details of the flow time-history. The moment coefficient has non-linear features that cannot be captured by retaining even the first 2 harmonics in the solution. The solutions including higher harmonics are in good agreement with each other and with the time-domain solution. Nevertheless, it is observed that the 3 mode HB result slightly overpredicts the moment coefficient during part of the downstroke motion. Experimental data ${ }^{21}$ are also included in Fig. 5. Deviations between numerical and experimental results are likely to be associated with the neglect of viscous forces and uncertainties in the experimental data. The time-domain and the HB COSA solutions are also included and compared against experimental data. Similar considerations to those given for the relative performance of the HB solver with respect to the respective time-domain method are observed for the COSA solutions.

Analyses of the frequency spectra of the integrated aerodynamic coefficients computed by the time-domain solution provide a way of interpreting these considerations, as shown in Fig. 6. Although of moderate magnitude, the force coefficient spectrum comprises the $2^{\text {nd }}$ harmonic. The frequency content of the moment coefficient is seen to extend up to the $4^{\text {th }}$ harmonic frequency. The magnitude of the $2^{\text {nd }}$ and $3^{\text {rd }}$ harmonics is $40 \%$ and $10 \%$, respectively, the magnitude of the fundamental harmonic. The magnitude of higher frequencies is limited to less than $5 \%$. 


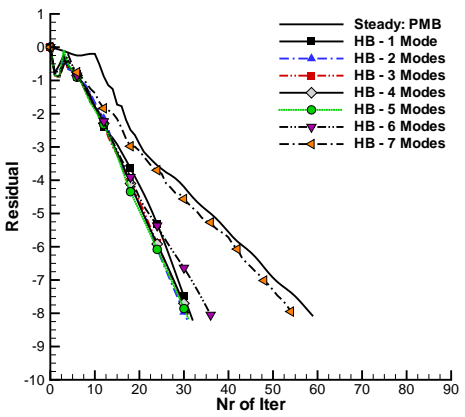

(a) AGARD CT2, PMB/HB solution

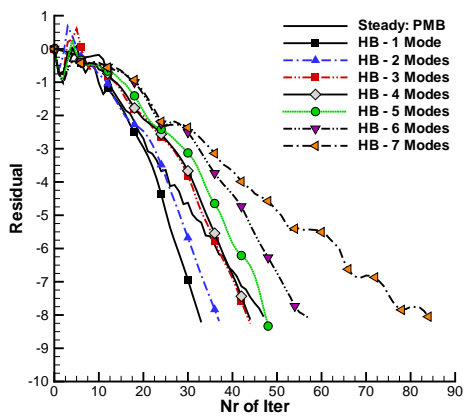

(d) AGARD CT5, PMB/HB solution

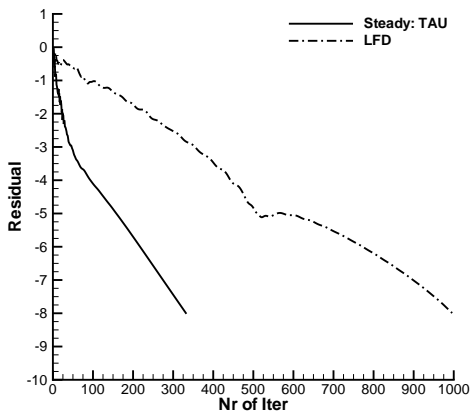

(b) AGARD CT2, TAU/LFD solution

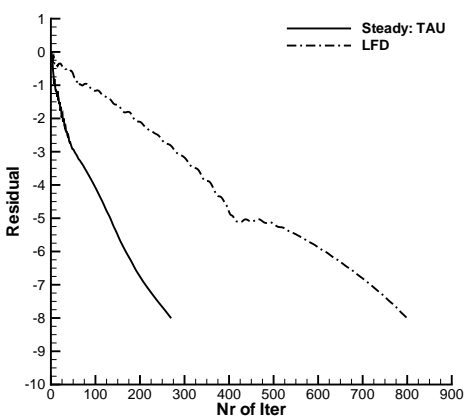

(e) AGARD CT5, TAU/LFD solution

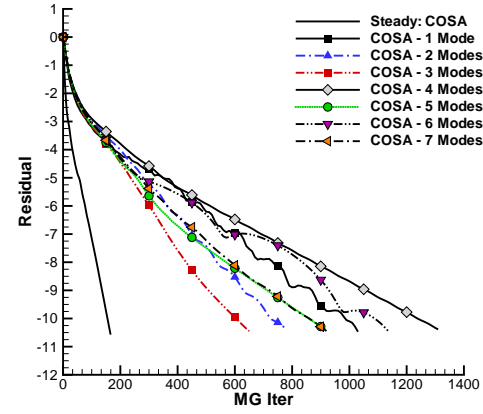

(c) AGARD CT2, COSA/HB COSA solution

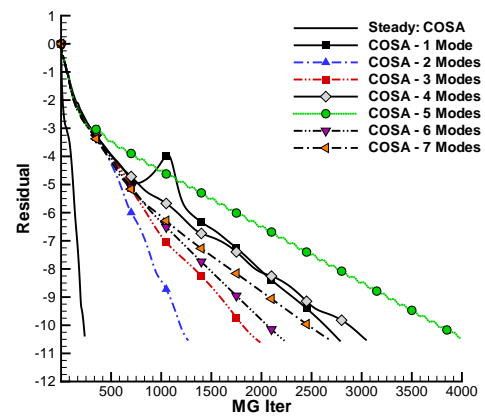

(f) AGARD CT5, COSA/HB COSA solution

Figure 4. Convergence of the L2 norm of the residual for the NACA 0012 test cases

Dynamic derivatives were calculated for all computational results and are summarized in Table 3 for the normal force and pitching moment coefficients. The values obtained from the time-domain solution are taken as reference values. Because the frequency content of the force coefficient time-history is dominated by the first 2 harmonics, the agreement in the prediction of the in-phase and out-of-phase components is excellent when 2 or more modes are retained in the solution. The spectrum of the moment coefficient contains 3 harmonics whose magnitude is larger than one tenth the magnitude of the fundamental harmonic, explaining the deviations in the prediction of dynamic derivatives for the solutions using less than 3 modes. The predicted values using 3 and 7 modes are in excellent agreement with each other and with the values from the time-domain solution. The percent error in the predicted values of dynamic derivatives using the HB solution with 3 modes is limited to less than $2 \%$ the reference values. For the normal force, the 1 mode HB and the LFD solution achieve a very similar and good agreement, instead the pitching moment could be resolved by the HB better, where the LFD is too far from any linear assumptions which can be seen furthermore in Fig. 5(b).

\begin{tabular}{lcccc}
\hline & $C_{N_{\alpha}}-k^{2} C_{N_{\dot{q}}}$ & $C_{N_{q}}+C_{N_{\dot{\alpha}}}$ & $C_{m_{\alpha}}-k^{2} C_{m_{\dot{q}}}$ & $C_{m_{q}}+C_{m_{\dot{\alpha}}}$ \\
\hline \hline Time Domain & 6.63 & -15.5 & 0.108 & -2.49 \\
HB - 1 mode & 6.67 & -13.1 & 0.146 & -3.41 \\
HB - 2 modes & 6.67 & -15.8 & 0.112 & -2.54 \\
HB - 3 modes & 6.65 & -15.8 & 0.105 & -2.49 \\
HB - 7 modes & 6.64 & -15.6 & 0.106 & -2.49 \\
LFD & 6.72 & -15.7 & 0.065 & -2.02 \\
\hline
\end{tabular}

Table 3. Test Case 1: dynamic derivatives for NACA 0012

The instantaneous pressure coefficient distribution from the numerical results at the mean angle of attack, which 


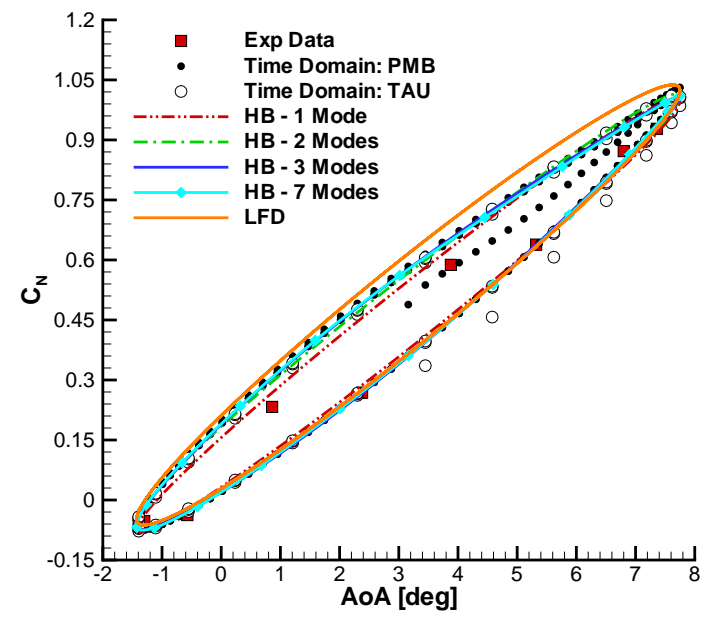

(a) Normal force coefficient, PMB and TAU

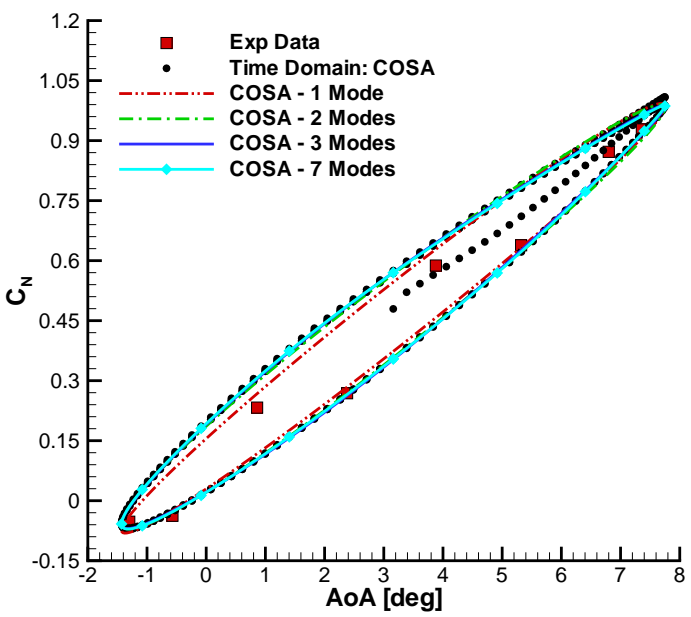

(c) Normal force coefficient, COSA

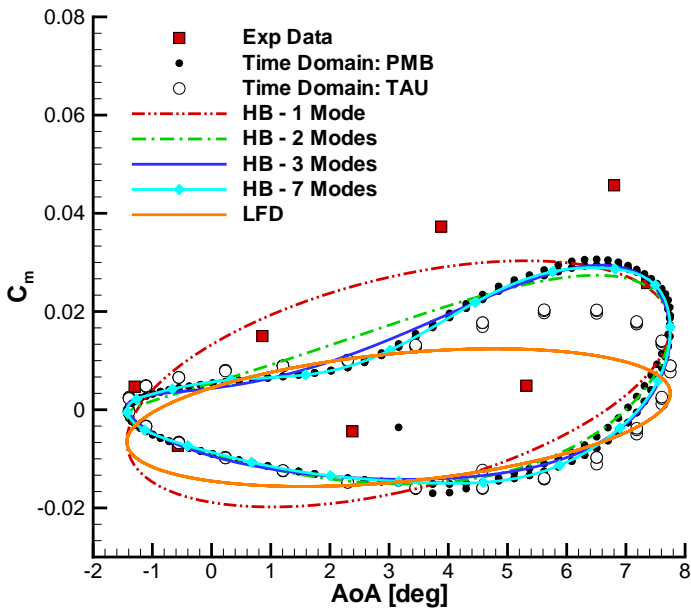

(b) Pitching moment coefficient, PMB and TAU

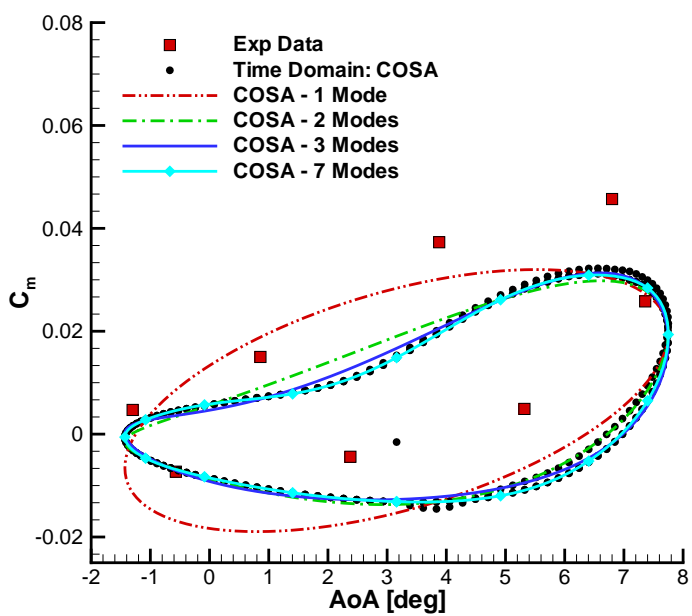

(d) Pitching moment coefficient, COSA

Figure 5. Test Case 1: normal force and pitching moment coefficient loops for NACA 0012

is included in the HB time intervals, for increasing angle was compared with experimental data from the AGARD CT2 case. The mean angle of attack is $\alpha_{0}=3.16^{\circ}$ and the nearest angle at which the pressure was measured is at $\alpha=2.38^{\circ}$ for increasing angle. In Fig. 7(a) the time-domain solution and the HB solutions obtained using 1, 2 and 3 modes are included. An excellent agreement is observed between the time-domain and the HB solutions, as well as with the experimental data. The reason the numerical solutions appear to overpredict the pressure coefficient on the upper surface, and underpredict on the lower side, is likely to be caused by the different instantaneous angle of attack available from the AGARD CT2 case. To investigate the effect of the number of modes on the accuracy of the solution, the mean values and the first harmonic of the unsteady surface pressure coefficient were computed from the numerical solutions. The solutions computed using 3 and 7 modes are in good agreement for the mean quantities on the lower surface, whereas the solutions computed using 1 and 2 modes miss some of the details of the flow on the upper side of the airfoil. This is shown in Fig. 7(b). For example, the location of the strong gradient in the pressure coefficient is predicted to be slightly further downstream for 1 and 2 harmonics. The peak value of pressure on the upper surface is also underpredicted for 1 harmonic. For the LFD result, the mean value of surface pressure coefficient is shown and represents the steady-state solution. In a steady flow, only a large suction region near the leading edge is identified 


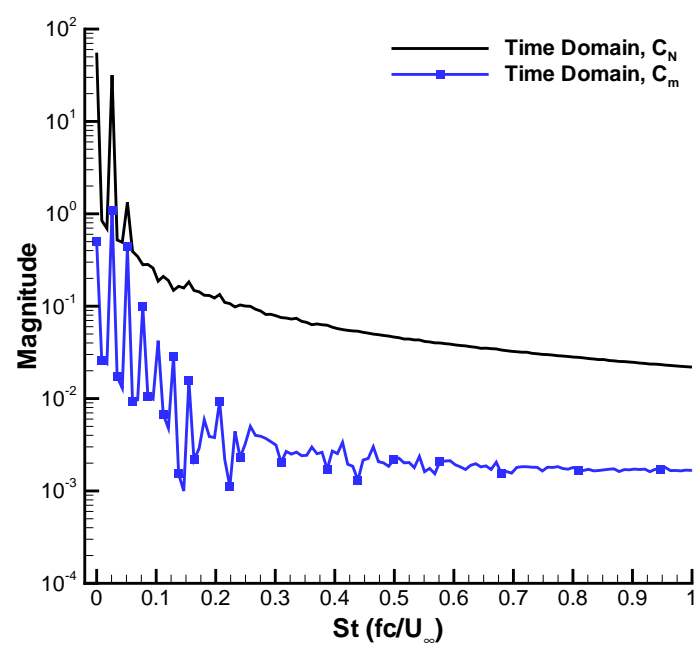

Figure 6. Test Case 1: magnitude of normal force and pitching moment coefficients for NACA 0012

and no shock wave is observed. The LFD solution is in agreement with the surface pressure coefficient distribution obtained in a steady state analysis using time-domain solvers. The HB solution is a time-average solution over the steady state analyses. It is recognized that the main difference between the LFD and the 1 mode HB solutions is that, at least, one of the $2 N_{H}+1$ steady state solutions for the HB method features a shock wave and the resulting mean value contains most of the non-linear features shown by time-domain methods. On the lower side in Fig. 7, the real and imaginary parts of the first harmonics are shown. The solutions obtained using 3 and 7 modes are in good agreement with the time-domain solution. For 3 harmonics, however, some of the details cannot be captured in the real part of the surface pressure distribution. Nevertheless, the agreement of the 3 modes solution with higher harmonics and the time-domain solution is within engineering accuracy. A poorer agreement is achieved by the LFD solution, which overpredicts the magnitude and underpredicts the width of the peaks. Similar inaccuracies were recently observed ${ }^{24}$ in the viscous simulation of transonic flow past oscillating flap on the NACA 0012. The first harmonic unsteady pressure coefficient using the LFD method represents the local solution calculated around the mean angle of attack for a small perturbation of the grid movement. For a small perturbation, the solution is linear with respect to the perturbation. A preliminary calculation was run with the time-domain methods for a small pitch amplitude and the corresponding first harmonic solution was in agreement with the LFD solution. The large peaks in the first harmonic components represent changes in the pressure coefficient distribution near the leading edge and are not connected with any moving shock wave. For this test case, no shock wave is predicted by the LFD solution.

Fig. 8 includes a set of results computed using the time-domain and the HB COSA methods. The overall performance of the HB method compared to the time-domain COSA solution is similar to that of the HB and the PMB solvers. Nonetheless, spurious oscillations around the shock wave are observed and are visible in the 7 modes HB solution. This feature may be due to some inaccuracies in the implementation of the flux limiter in COSA, and the matter is currently being investigated.

\section{Test Case 2: AGARD CT5}

For the AGARD CT5, the flow solution is non-linear, with a shock appearing in the leading edge region and moving downstream. The shock continues downstream until approximately $45 \%$ of the chord. Then the shock returns upstream close to the leading edge. The same pattern is repeated on the opposite side of the airfoil. The flow remains attached throughout the cycle of unsteadiness. Since this case features a strong shock on the upper and lower surface, the question is whether the presence of the shock has a negative impact on the accuracy of the time-linearized code.

The normal force and the pitching moment coefficients referred to body axes are considered for the comparison of the HB and the LFD solvers with the time-accurate solutions. The loops of the integrated aerodynamic coefficients against the instantaneous angle of attack are shown in Fig. 9. The HB solution was obtained using 1, 2, 3 and 7 


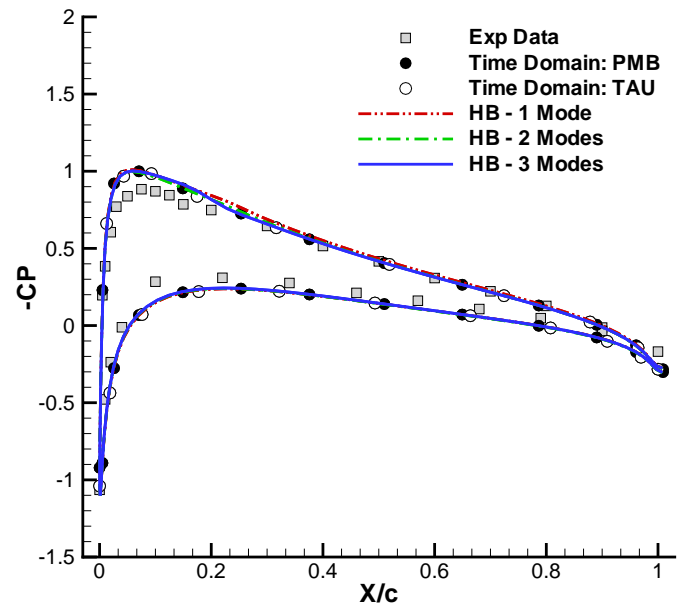

(a) Instantaneous pressure, $\alpha=3.16^{\circ}$ (up)

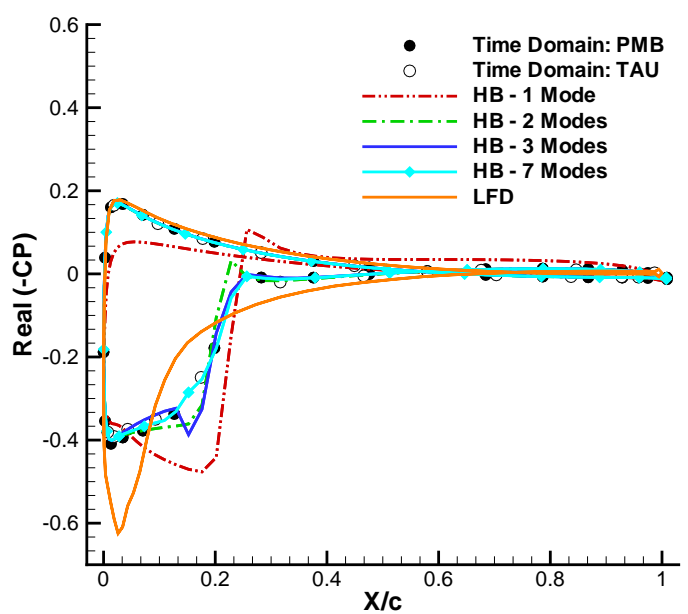

(c) Real part, $1^{\text {st }}$ harmonic

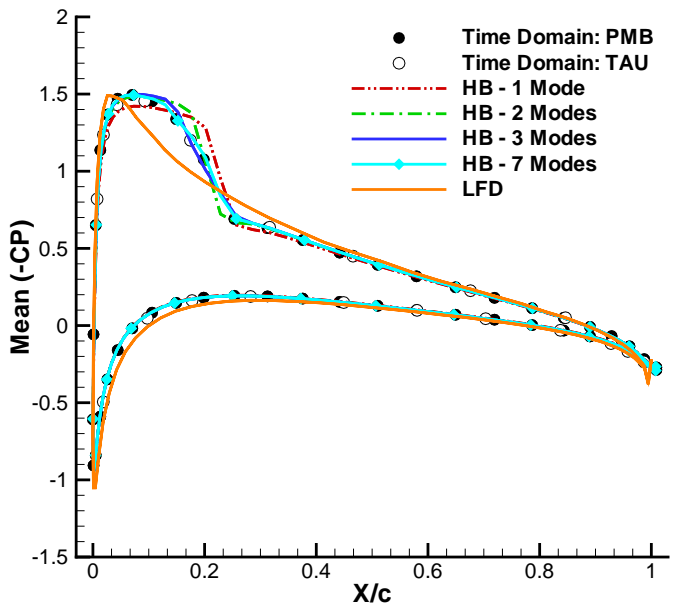

(b) Mean value

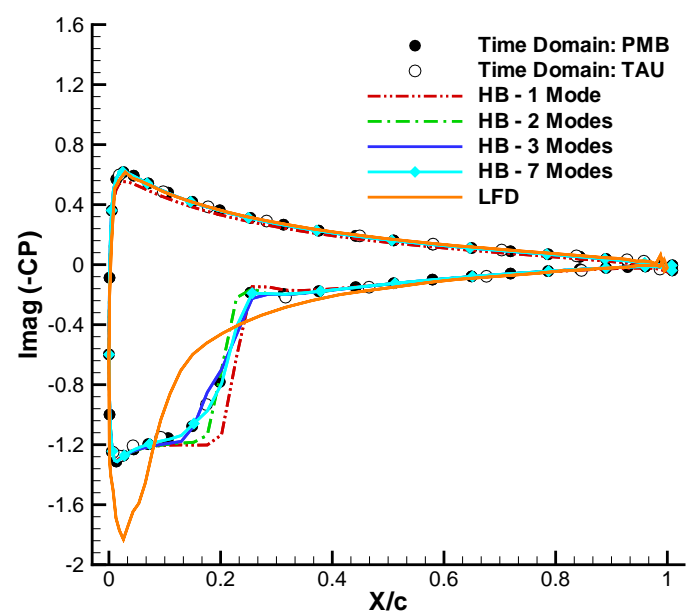

(d) Imaginary part, $1^{\text {st }}$ harmonic

Figure 7. Test Case 1: pressure coefficient distribution for NACA 0012; solutions are included for the PMB/HB and TAU/LFD solvers; the term up in parenthesis indicates the direction increasing angle; experimental data were measured at $\alpha=2.38^{\circ}$ for increasing angle

modes. The solutions computed using 1,2, 3 and 7 modes agree well in predicting the force loop. The LFD solution of the lift force coefficient shows good agreement in comparison to the time-accurate TAU simulation. The moment coefficient has non-linear features because of the moving shock wave that cannot be captured by retaining the first 2 harmonics in the solution. The LFD and the HB results for the first harmonic shows that both solutions will not be able to give accurate predictions. Furthermore, both pitching moment curves indicate a phase shift to the timeaccurate solutions. The solutions computed using higher harmonics are in good agreement with each other and with the time-domain solution. Nevertheless, it is observed that the 3 modes HB result slightly misses some of the flow features at the highest and lowest angles of attack. The HB COSA solutions are in agreement with the respective time-domain method increasing the number of modes and, in particular, have a similar performance to that of the HB solver. Furthermore, the initial transitory in the time-accurate solution from the steady state analysis is nearly identical for the PMB and COSA methods.

Analyses of the frequency spectra of the integrated aerodynamic coefficients provide a way of interpreting these observations, as shown in Fig. 10. The frequency spectrum of the force coefficient, as expected by the excellent 


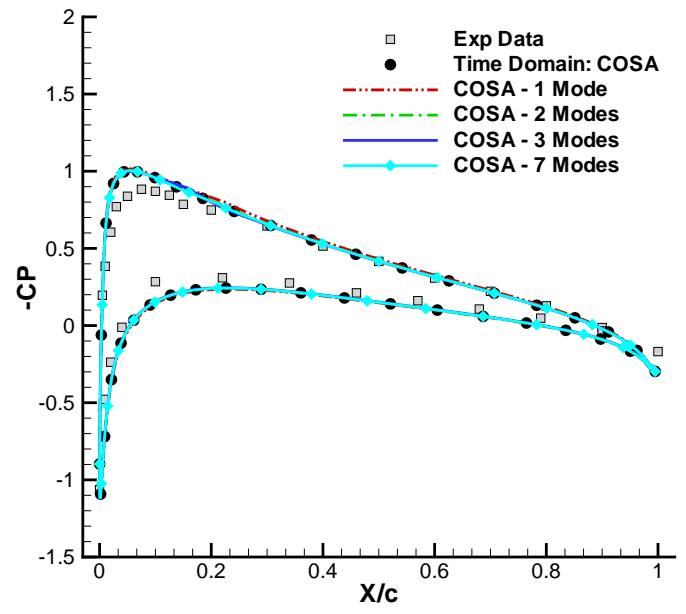

(a) Instantaneous pressure, $\alpha=3.16^{\circ}$ (up)

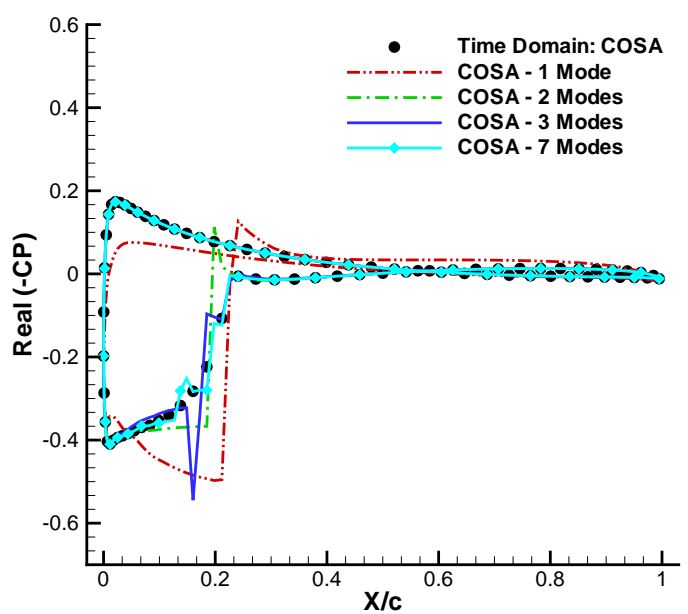

(c) Real part, $1^{\text {st }}$ harmonic

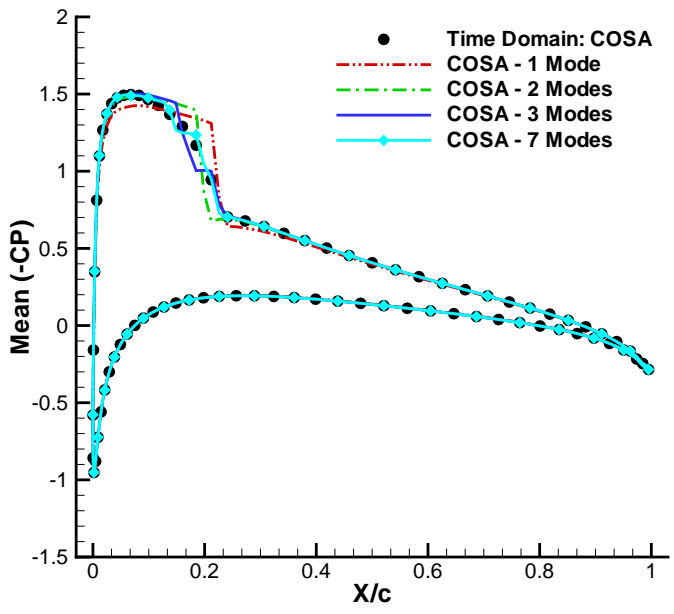

(b) Mean value

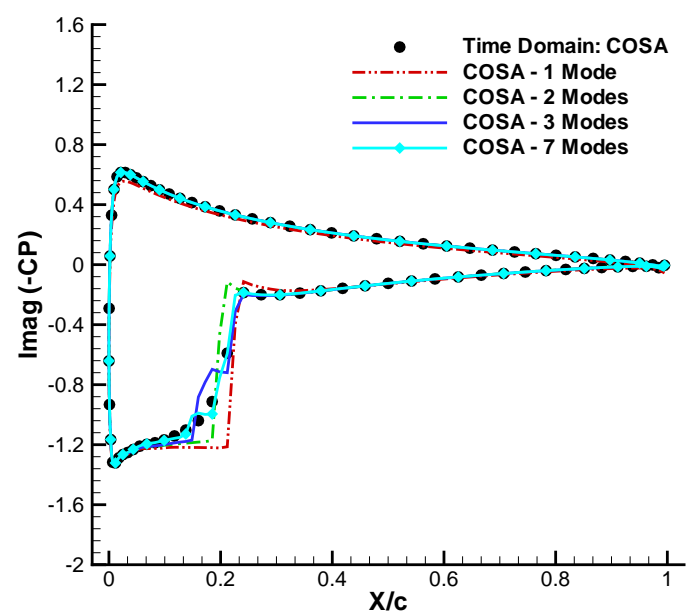

(d) Imaginary part, $1^{\text {st }}$ harmonic

Figure 8. Test Case 1: pressure coefficient distribution for NACA 0012; solution is included for the time-domain and HB COSA solvers; the term up in parenthesis indicates the direction increasing angle; experimental data were measured at $\alpha=2.38^{\circ}$ for increasing angle

matching for the first harmonic, is defined solely by the fundamental harmonic. The frequency band of the moment coefficient extends up to the $5^{\text {th }}$ harmonic. However, one can see that the amplitude of the $2^{\text {nd }}$ harmonic is far lower than the amplitude of the $3^{\text {rd }}$ harmonic. Similarly, the amplitude of any odd harmonic is lower than the amplitude of the corresponding even harmonic at higher frequencies. Reconsidering Fig. 9, it is observed that, apart from a small adjustment in the mean slope, no significant improvements are achieved between the HB solutions using 1 and 2 modes in the prediction of the moment loop. However, when considering the $3^{\text {rd }}$ mode the solution contains most of the non-linear features of the time-domain solution, achieving a good overall agreement. The reasons behind this observed feature are not clear yet; nonetheless, the non-linearities in the moment coefficient at approximately 1/7 and $2 / 3$ of one period seem to be related to the shock wave moving upstream toward the leading-edge and the forming shock wave moving downstream toward mid-chord.

Dynamic derivatives were calculated for all computational results and are summarized in Table 4 for the normal force and pitching moment coefficients. The values obtained from the time-domain solution are taken as reference values. Because the frequency content of the force coefficient time-history is characterised by the $1^{\text {st }}$ harmonic only, 


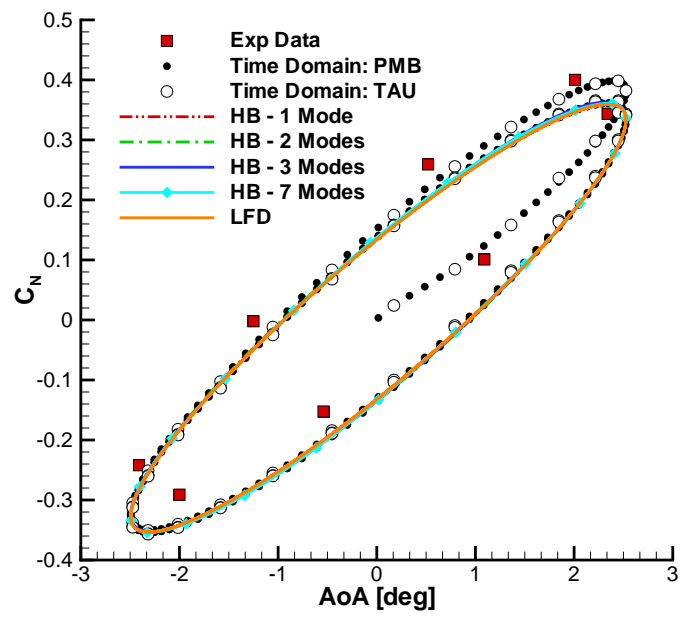

(a) Normal force coefficient, PMB and TAU

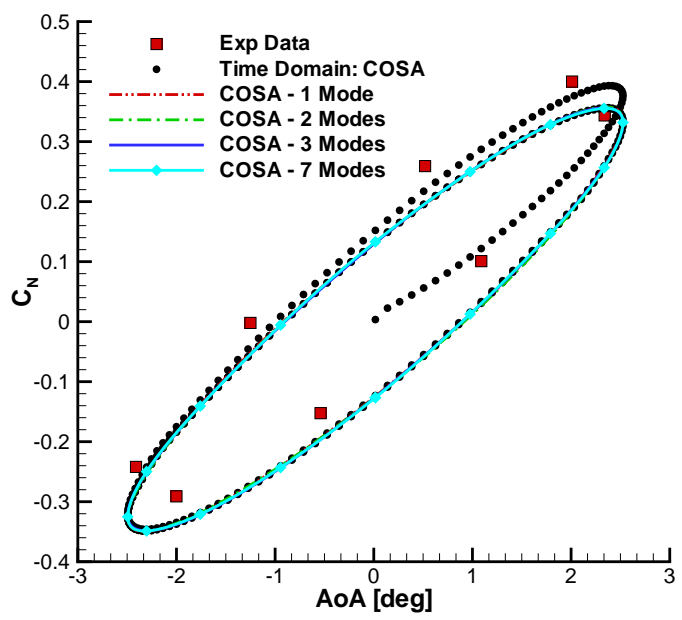

(c) Normal force coefficient, COSA

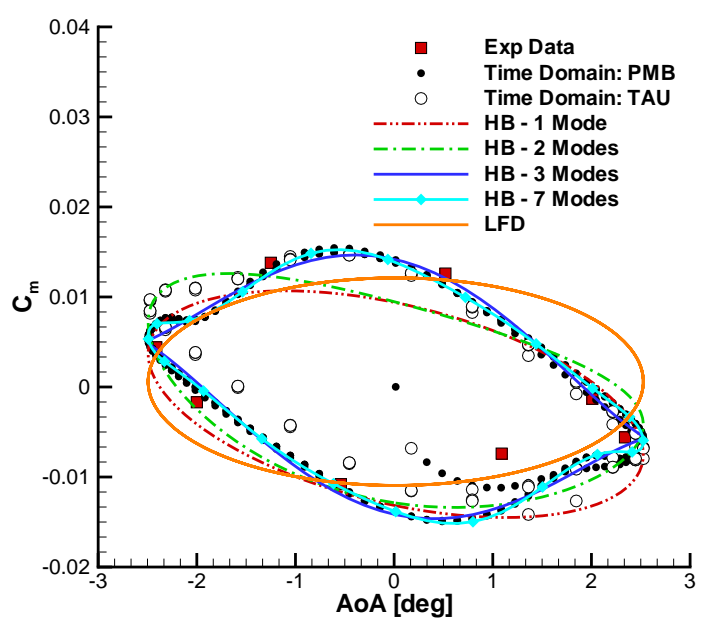

(b) Pitching moment coefficient, PMB and TAU

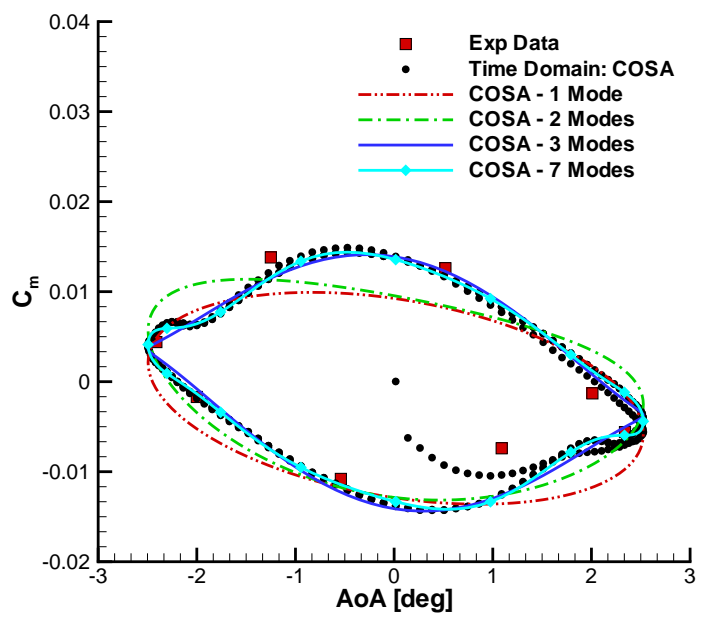

(d) Pitching moment coefficient, COSA

Figure 9. Test Case 2: normal force and pitching moment coefficient loops for NACA 0012

the agreement in the prediction of the in-phase and out-of-phase components is excellent regardless of the number of modes. Although the spectrum of the moment coefficient included more harmonics whose magnitude was larger than one tenth the magnitude of the fundamental harmonic and the flow is transonic, the agreement is good. The predicted values using 2, 3 and 7 modes are in excellent agreement with each other and with the values from the timedomain solution. The percent error in the predicted values of dynamic derivatives using the HB solution, regardless of the number of modes, is limited to less than $2 \%$ of the reference values. The predictions of the LFD consist of a reasonable value for the out-of-phase component, while the in-phase component highlights a large inaccuracy.

The instantaneous pressure coefficient distribution from the numerical results at the mean angle of attack, which is included in the HB time intervals, for increasing angle was compared with experimental data from the AGARD CT5 case. The mean angle of attack is $\alpha_{0}=0.016^{\circ}$ and the nearest angle at which the pressure was measured is at $\alpha=-0.54^{\circ}$ for increasing angle. In Fig. 11(a) the time-domain solution and the HB solutions obtained using 1, 2 and 3 modes are included. An excellent agreement is observed between the time-domain and the HB solutions, as well as with the experimental data. However, the location of the shock wave is predicted to be slightly further upstream for one harmonic. The mean values and the first harmonic of the unsteady surface pressure coefficient were computed from the 


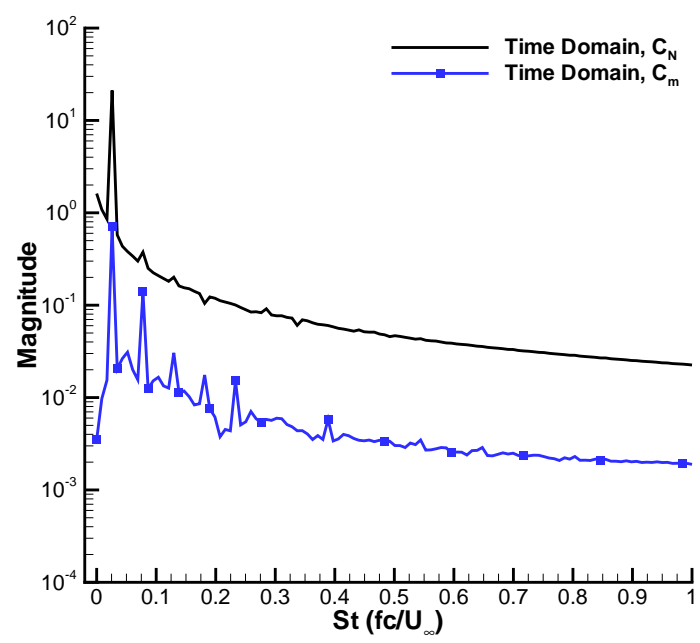

Figure 10. Test Case 2: magnitude of normal force and pitching moment coefficients for NACA 0012

\begin{tabular}{lcccc}
\hline & $C_{N_{\alpha}}-k^{2} C_{N_{\dot{q}}}$ & $C_{N_{q}}+C_{N_{\dot{\alpha}}}$ & $C_{m_{\alpha}}-k^{2} C_{m_{\dot{q}}}$ & $C_{m_{q}}+C_{m_{\dot{\alpha}}}$ \\
\hline \hline Time Domain & 7.58 & -37.8 & -0.127 & -3.09 \\
HB - 1 mode & 7.55 & -37.7 & -0.128 & -3.16 \\
HB - 2 modes & 7.56 & -38.2 & -0.130 & -3.13 \\
HB - 3 modes & 7.57 & -38.1 & -0.126 & -3.10 \\
HB - 7 modes & 7.57 & -38.1 & -0.127 & -3.11 \\
LFD & 7.55 & -30.4 & 0.005 & -3.24 \\
\hline
\end{tabular}

Table 4. Test Case 2: dynamic derivatives for NACA 0012

numerical solutions. The solutions computed using 2, 3 and 7 modes are in good agreement for the mean quantities, whilst the solution computed using 1 mode misses important details near the shock wave location. An important remark is for the different shock pattern on the upper and lower surfaces for the 1 mode HB solution, which is not seen otherwise using a larger harmonic base or time-domain method. The reason for overpredicting the shock strength on the lower side and underpredicting it on the upper surface for the 1 mode HB solution is found on the $2 N_{H}+1$ snapshots included in the HB method which are a solution base sampled at uniformly spaced temporal points. It is observed that 2 snapshots feature a shock wave on the lower side and the remaining snapshot on the upper surface, resulting in the mean value shown in Fig. 11(a). Fig. 11(b) presents the mean pressure distribution and additionally the initial steady state solution used for the LFD solver. In the sonic and transonic regions of the flow, the mean surface pressure is in good agreement with the HB and time-domain solutions. However, the pressure coefficient peak across the shock is not resolved good, being distributed over a wider chordwise location while the steady state distribution for the LFD method displays no shock wave which is included in a ordinary steady state analysis using time-domain methods. A reason for the unexpected poor agreement in the mean value can be the use of a too coarse grid not adapted to accurately resolve a shock wave, although the time-domain TAU solution is in agreement with the PMB result. The real and imaginary parts of the first harmonics are also shown in Fig. 11(c) and Fig. 11(d). The solutions obtained using 3 and 7 modes are in agreement with the time-domain solution. However, for 3 harmonics oscillations can be seen slightly further upstream of the shock wave location. For the LFD solution, similar considerations to those given for the AGARD CT2 case can be drawn. The large gradients in the pressure coefficient are associated with a moving shock on the airfoil surface because the steady state solution features a flow discontinuity.

Fig. 12 shows the solution using the time-domain and the HB COSA solvers. For the snapshot of instantaneous pressure coefficient, similar considerations to those given for the HB and PMB solvers are valid. It is noted that the 
time-domain COSA method predicts a stronger shock wave, possibly due to the better mesh refinement. As more Fourier modes are retained in the solution, convergence to the time-domain results is achieved. It is not possible yet to identify the reasons of spurious oscillations around the shock wave when 7 complex harmonics are included in the HB COSA solution. As previously explained, the spurious oscillations around the shock are possibly due to some aspects of the implementation of the flux limiter which are currently being investigated.

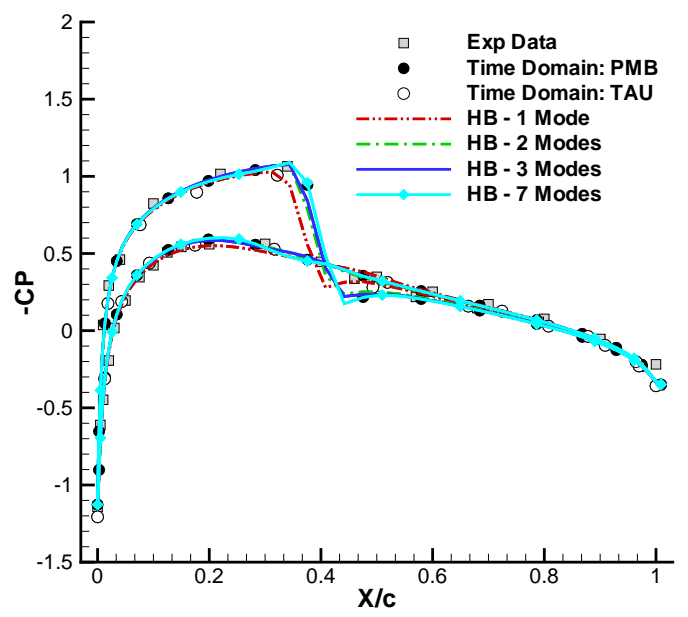

(a) Instantaneous pressure, $\alpha=0.016^{\circ}$ (up)

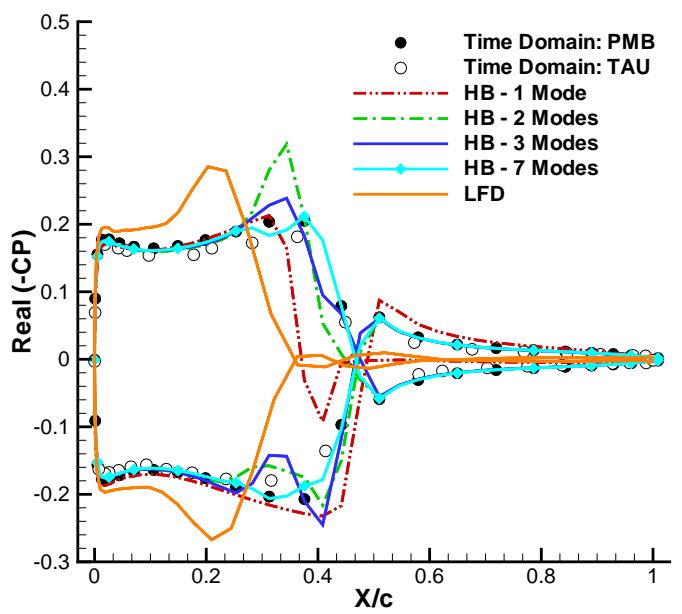

(c) Real part of $1^{\text {st }}$ harmonic

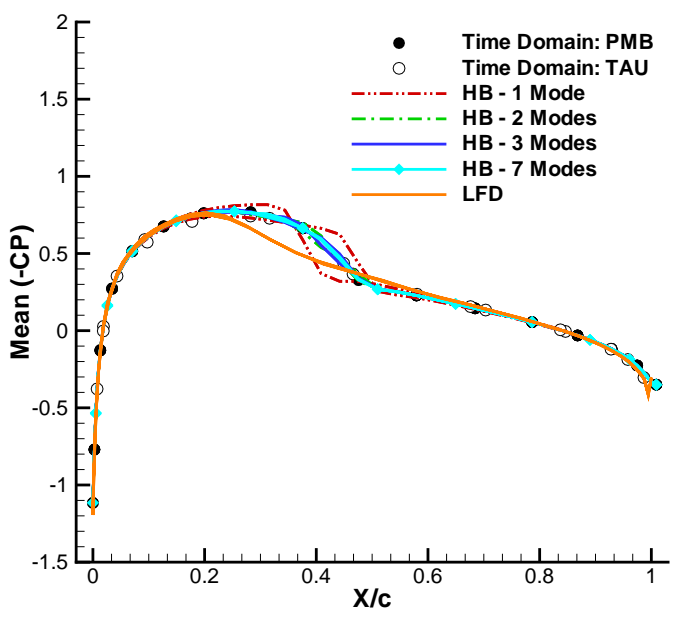

(b) Mean value

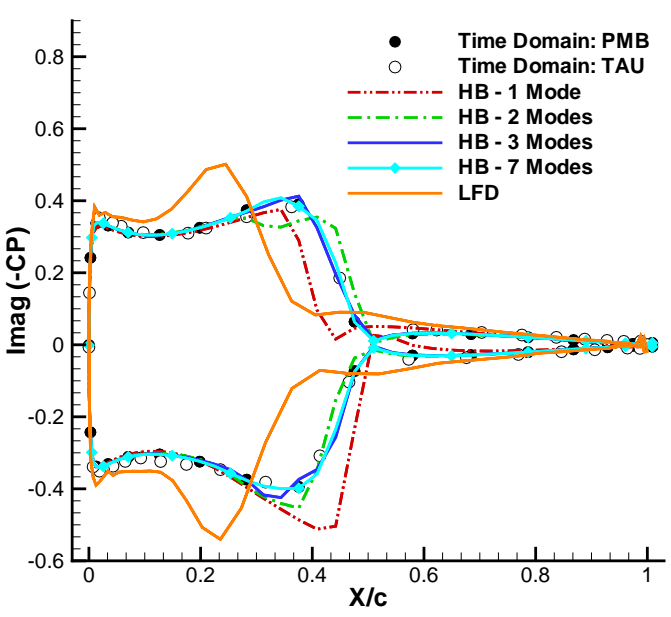

(d) Imaginary part of $1^{\text {st }}$ harmonic

Figure 11. Test Case 2: pressure coefficient distribution for NACA 0012; solutions are included for the PMB/HB and TAU/LFD solvers; the term up in parenthesis indicates the direction increasing angle; experimental data were measured at $\alpha=-0.54^{\circ}$ for increasing angle

\section{Computational Efficiency and Overall Performance}

Fig. 13 shows the speed up of the HB, LFD and HB COSA solvers compared to the respective time-domain methods as a function of the number of Fourier modes. For the LFD method, the run times for the two cases are nearly identical achieving a speed up of about 10. A similar performance is obtained by the 1 mode HB solver, with a limited loss of efficiency for the AGARD CT5 case for which the flow physics is more complex. As more Fourier modes are retained in the solution, the run times grow exponentially. Above 5 harmonics, the HB solutions result more computationally expensive than the solution obtained by the time-domain solver. Timings for the acceleration techniques are only 


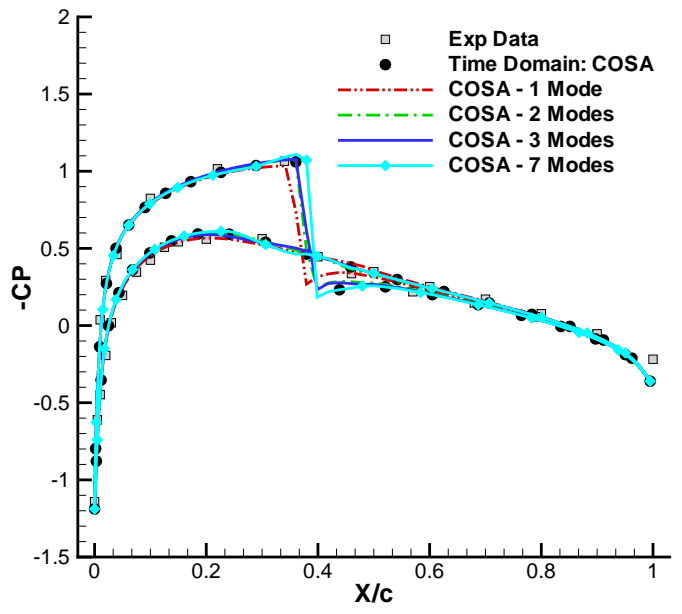

(a) Instantaneous pressure, $\alpha=0.016^{\circ}$ (up)

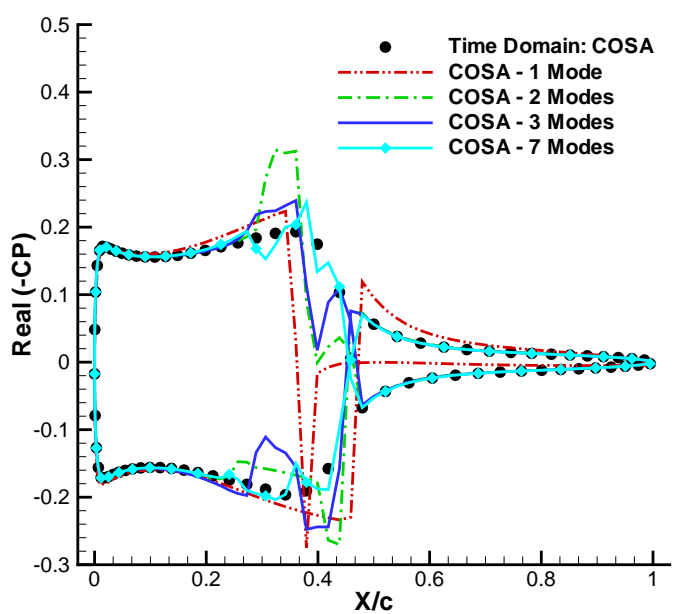

(c) Real part of $1^{\text {st }}$ harmonic

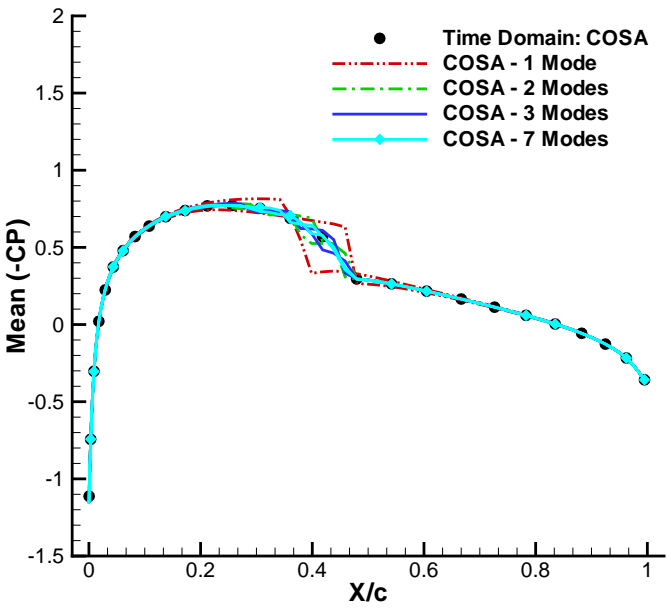

(b) Mean value

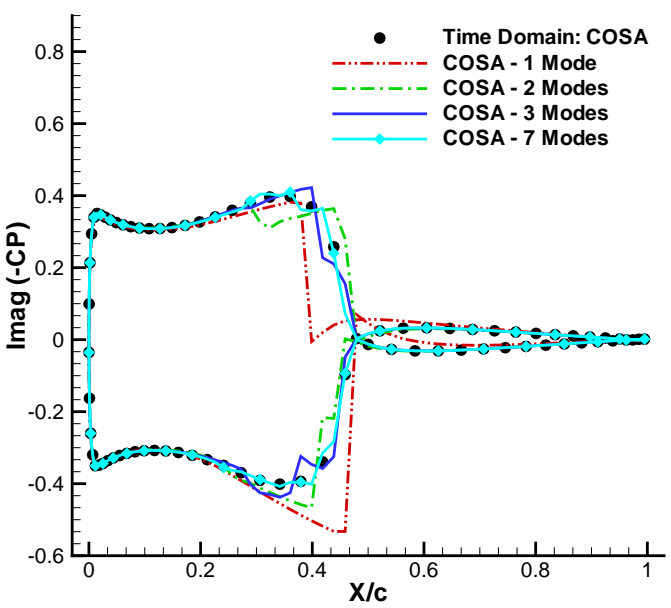

(d) Imaginary part of $1^{s t}$ harmonic

Figure 12. Test Case 2: pressure coefficient distribution for NACA 0012; solution is included for the time-domain and HB COSA solvers; the term up in parenthesis indicates the direction increasing angle; experimental data were measured at $\alpha=-0.54^{\circ}$ for increasing angle

indicative and numerical parameters were not optimized for a best performance. It was mentioned that for the HB solver a local adaptive CFL number procedure was adopted. For large CFL numbers, the increase in CPU time was caused by the degradation of the preconditioner as the time step was increased. To avoid this negative issue, the CFL number was limited up to a maximum value. The maximum CFL number was progressively reduced as more harmonics were retained in the solution because of the increased time for the evaluation of each pseudo iteration.

Table 5 summarizes for the multigrid solver convergence informations for the Test Case 1 and 2. The variable labelled Relative $C P U$ is the ratio between a single MG iteration per elemental harmonic in the framework of the $\mathrm{HB}$ solution and that required for a single MG iteration in the framework of the time-domain solution. The former is obtained by dividing the overall CPU-time needed for the HB analysis under consideration by the overall number of multigrid iterations and $2 N_{H}+1$, where $N_{H}$ is the number of complex harmonics.

The first comment on the data reported is that the CFL number could be kept at the relatively high value of 3 for both the time-domain and the HB analyses for Test Case 1 . The physically more complex flow associated with Test Case 2 , however, required the use of lower CFL numbers for both time-domain and HB analyses. More specifically, the CFL 
number of the HB analyses had to be lowered to 1 in order to maintain numerical stability. The CFL number used for the time-domain analysis could instead be 2 . Indeed the time-domain analysis of Test Case 2 could be performed also with a CFL of 3, but in this circumstance the maximum residual failed to converge to $10^{-10}$ in less than 1000 multigrid iterations for a small number of physical times. The second important remark is that for both test cases the number of multigrid cycles required to achieve the prescribed convergence level of $10^{-10}$ did not increase monotonically with the number of harmonics used in the analysis. A conclusive explanation for this behavior cannot be provided yet. Asymptotic spectral analysis of the preconditioned HB multigrid operator for problems with different types and levels of nonlinearities solved by using different number of harmonics may shed some light on this aspect. The cost of the $\mathrm{HB}$ multigrid interation increases with respect to that of the time-domain multigrid iteration as the number of complex harmonics increases. This is because of the cost associated with the matrix-vector product required to compute the first term of the left-hand-side of Eq. (8). Such a cost clearly increases with the number of complex harmonics.

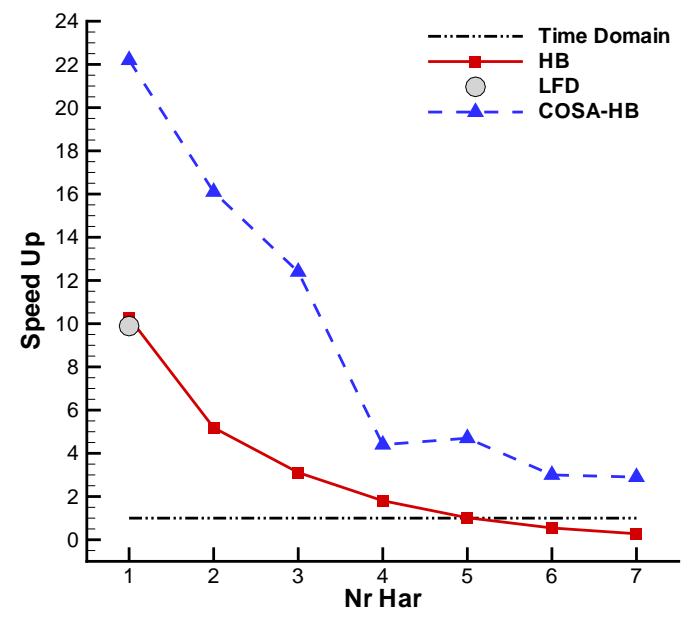

(a) Test Case 1 - AGARD CT2

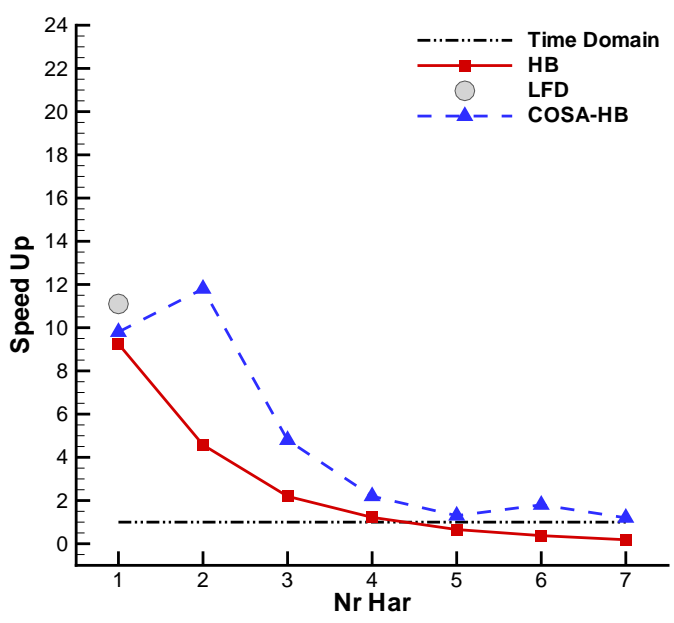

(b) Test Case 2 - AGARD CT5

Figure 13. CPU time speed up for the HB, LFD and HB-COSA methods compared with respective time-domain methods for the NACA 0012 test cases

\begin{tabular}{lccccccc} 
& \multicolumn{7}{c}{ Nr of Harmonics } \\
& 1 & 2 & 3 & 4 & 5 & 6 & 7 \\
\cline { 2 - 8 } AGARD CT 2 & \multicolumn{10}{c}{ (1030 } & 780 & 650 & 1310 & 920 & 1140 & 920 \\
\hline MG cycles & 1.23 & 1.35 & 1.50 & 1.61 & 1.76 & 1.87 & 2.08 \\
Relative CPU & 1.070 & \\
\hline \hline AGARD CT 5 & & & & & & & \\
\hline MG cycles & 2790 & 1270 & 1990 & 3050 & 4050 & 2230 & 2690 \\
Relative CPU & 1.24 & 1.37 & 1.52 & 1.65 & 1.79 & 1.94 & 2.13 \\
\hline \hline
\end{tabular}

Table 5. Convergence informations for the HB COSA method for the NACA 0012 test cases 


\section{B. Standard Dynamics Model Aircraft}

The Standard Dynamics Model (SDM) is a generic fighter configuration based loosely on the F-16 planform. The model includes a slender strake-delta wing, horizontal and vertical stabilizers, ventral fin and a blocked off inlet. This geometry has been used at various wind tunnel facilities to collect data using different measurement techniques. ${ }^{25-28}$ Further details on the geometry can be found in Huang. ${ }^{29}$

This model has been used for two previous studies related to dynamic derivatives. First, the development of a flight dynamics aerodynamic model using CFD was reported in Ghoreyshi et al. ${ }^{30}$ Forced manoeuvres were used to evaluate the applicability of the dynamic derivative model. The results of a study that considers the variation of the dynamic derivatives with various aerodynamic and motion parameters, based on time domain simulations is reported in Da Ronch et al. ${ }^{20}$

A block structured mesh was generated for the use with the PMB and HB solvers and it is shown in Fig. 14 along with the unstructured mesh for the TAU and LFD solvers. The geometry was slightly simplified by removing the blocked off intake and the ventral fins. The structured mesh was obtained with 701 thousand points. The unstructured grid consists of 59542 points, 310156 tetrahedra and 27246 surface triangles. The COSA solvers were not run for this configuration.

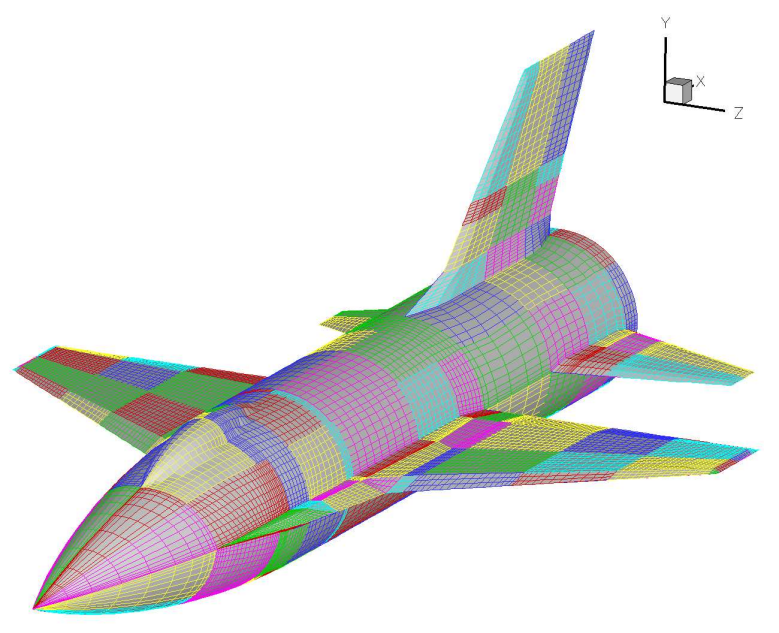

(a) Block structured grid for the PMB and HB solvers

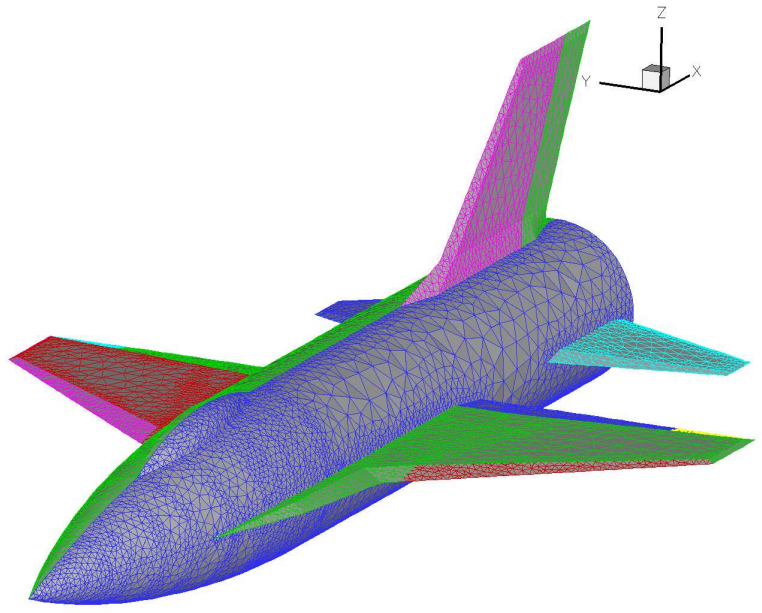

(b) Unstructured grid for the TAU and LFD solvers

Figure 14. Surface grid for the SDM model geometry

To assess the use of the HB, LFD and time-domain methods, a subsonic test case at low angles of incidence is first considered. The Mach number is 0.3 and the mean angle of attack is $0.0^{\circ}$. A numerical study was carried out to evaluate the influence of the amplitude of motion on the aerodynamic loads for amplitudes from $2.0^{\circ}$ up to $5.0^{\circ}$. It was observed that the motion with an amplitude of $2.0^{\circ}$ ensures a virtually linear aerodynamic response. Works by the co-authors ${ }^{20,30}$ have pinpointed the presence of vortical features in the flow field at $15.0^{\circ}$ angle of incidence. The superposition of a pitching motion on the background state results in the periodic motion of the vortices, stressing the non-linearities of the flow solution. Demonstration of the non-linear capabilities of the HB method in capturing the aerodynamic frequencies excited by the harmonic boundary conditions movement is presented. Experimental ${ }^{31}$ and numerical ${ }^{32}$ investigations of the damping in pitch derivative through the transonic regime were conducted. Assessment of the numerical techniques is performed for Mach numbers between 0.4 up to 1.1 at $0.0^{\circ}$ angle of incidence, with a finer step increment near Mach 1 where a significant change in damping value was observed. The reduced frequency was constant, so that an increment in the freestream speed was followed by a proportional increment in the frequency of motion. With a flowfield featuring the formation of shock waves and their time-dependent motion, a time step study was undertaken to evaluate the influence of the time resolution on the flow solution. It is demonstrated that 20 time steps per pitching cycle are adequate for these cases for time-domain methods. ${ }^{20}$

The flow conditions for all test cases are summarized in Table 6 . The moment reference point and the rotation point are located at the centre of gravity. The choice of main numerical parameters guarantees well converged analyses for all cases. A first remark is that the CFL number used for unstructured solvers was 1.2 for all cases. For the PMB and 
HB solvers, it is held constant to 100 for all cases whose Mach number was lower than 0.9 , for the remaining analyses lowered to 15 .

\begin{tabular}{llll}
\hline \hline & Test Case 1 & Test Case 2 & Test Case 3 \\
\hline Mach number, $M$ & 0.3 & 0.3 & 0.4 up to 1.1 \\
Mean incidence, $\alpha_{0}$ & $0.0^{\circ}$ & $15.0^{\circ}$ & $0.0^{\circ}$ \\
Pitch amplitude, $\alpha_{A}$ & $2.0^{\circ}$ & $5.0^{\circ}$ & $5.0^{\circ}$ \\
Reduced frequency, $k$ & 0.0493 & 0.0493 & 0.0493 \\
\hline \hline
\end{tabular}

Table 6. Description of the SDM test cases

\section{Numerical Results}

\section{Test Case 1: Subsonic}

The test case is run at low speed and, because of the small amplitude of motion, the flow is linear or quasi-linear. The aerodynamic loops for the time-domain solvers and the HB and the LFD methods are included in Fig. 15 for the normal force and pitching moment coefficient. The PMB and the TAU solutions predict linear responses to the input motion. The HB results match closely the PMB solutions for both force and moment coefficients. Most importantly, the results exhibit little variation over the range of Fourier modes and one time varying mode provides a solution convergent to plotting accuracy.

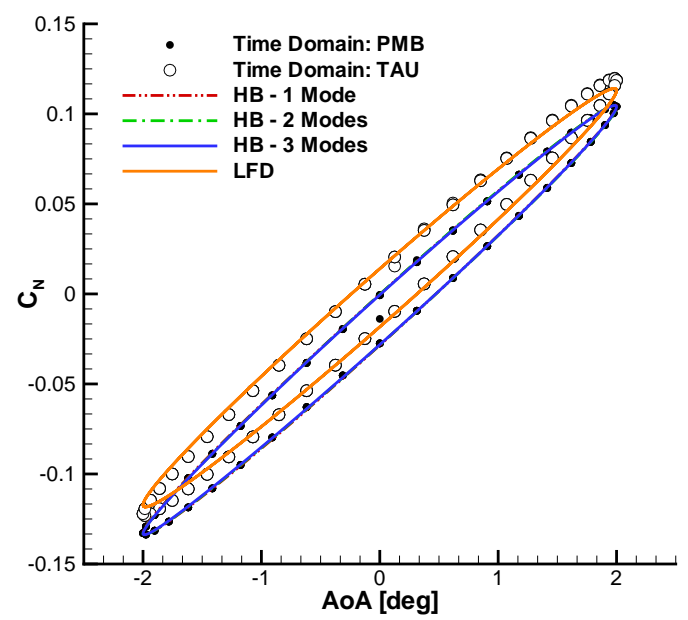

(a) Normal force coefficient

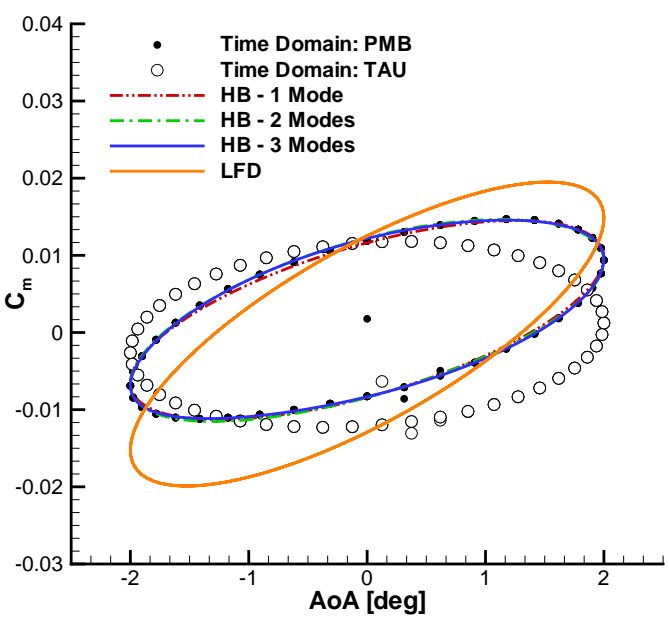

(b) Pitching moment coefficient

Figure 15. Test Case 1: normal force and pitching moment coefficient loops for the SDM model geometry

Table 7 summarizes the dynamic derivatives for the force and moment coefficients for the time-domain, HB and LFD solutions. The 1 mode HB results agree well with the PMB dynamic derivatives and convergence to the timedomain values is observed increasing the number of modes. Inaccuracies for the LFD solution are seen for the moment coefficient.

Test Case 2: Subsonic with vortical flow

At low speed and $15.0^{\circ}$ mean angle of attack, strake and wing vortices develop and interact. It was found that the amplitude of the sinusoidal motion has critical influence on the aerodynamic loops. Studies based on the PMB solver were carried out for amplitudes of $2.0^{\circ}, 3.5^{\circ}$ and $5.0^{\circ}$. While the aerodynamic loops had an ellipse-like shape for the smallest amplitude, non-linearities in the loops appeared for $3.5^{\circ}$ amplitude. The case with an amplitude $5.0^{\circ}$ is used to assess the validity and limitations of the non-linear HB method. The LFD solver was not run for this case because of vortical flow developments and vortices dynamics which are beyond the assumptions of the method. 


\begin{tabular}{lcccc}
\hline & $C_{N_{\alpha}}-k^{2} C_{N_{\dot{q}}}$ & $C_{N_{q}}+C_{N_{\dot{\alpha}}}$ & $C_{m_{\alpha}}-k^{2} C_{m_{\dot{q}}}$ & $C_{m_{q}}+C_{m_{\dot{\alpha}}}$ \\
\hline \hline Time Domain: PMB & 3.39 & 7.90 & 0.23 & -5.95 \\
HB - 1 mode & 3.39 & 8.20 & 0.23 & -5.82 \\
HB - 2 modes & 3.39 & 8.16 & 0.23 & -6.01 \\
HB - 3 modes & 3.39 & 7.99 & 0.23 & -5.97 \\
Time Domain: TAU & 3.44 & 10.99 & 0.07 & -6.81 \\
LFD & 3.30 & 9.38 & 0.43 & -7.39 \\
\hline
\end{tabular}

Table 7. Test Case 1: dynamic derivatives for the SDM model geometry

The normal force and the pitching moment coefficients against the instantaneous angle of attack are included in Fig. 16. The HB solutions, including up to 4 harmonics, are compared with the respective time-domain solutions. For the HB solver, the solutions using 3 and 4 harmonics agree well in predicting the force loop. However, both of these results fail to represent the inflection in force coefficient during the upstroke motion, evident at the instantaneous angle of attack of $16.0^{\circ}$. This consideration suggests that the force coefficient spectrum might contain an harmonic component that is not modeled by the 4 modes HB solution, thereby outside of this frequency range. Consistent differences can be noted for the pitching moment coefficient. A characteristic shape of "eight" at around $18.0^{\circ}$ appeared. The solutions using 1 and 2 harmonics achieve a poorer agreement in predicting the moment loop. However, the solutions retaining 3 and 4 harmonics predict well the main features including the shape of "eight".

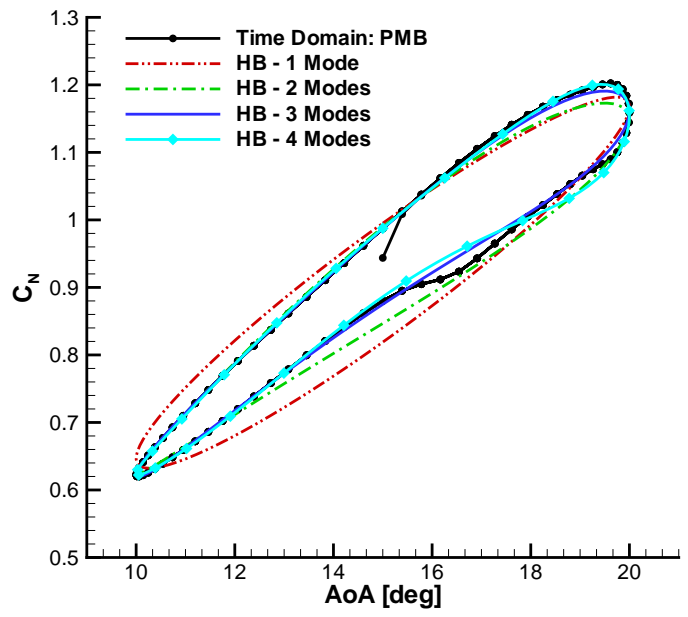

(a) Normal force coefficient

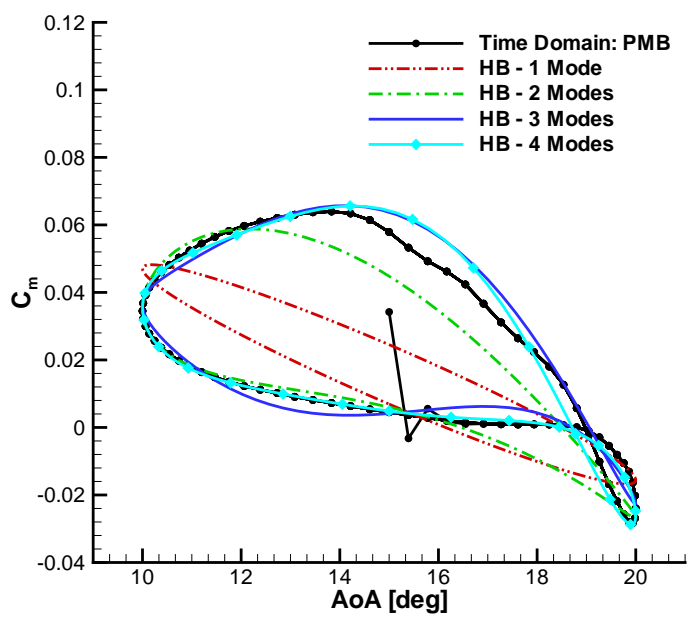

(b) Pitching moment coefficient

Figure 16. Test Case 2: normal force and pitching moment coefficient loops for the SDM model geometry

Fig. 17 shows the frequency spectra of the normal force and pitching moment coefficients computed by the timedomain solutions. It is observed that the normal force frequency spectrum contains the $2^{\text {nd }}$ and $3^{r d}$ harmonics, whose amplitude is $11 \%$ and $3 \%$, respectively, the amplitude of the fundamental harmonic. While the $4^{\text {th }}$ and $5^{\text {th }}$ harmonics are less than one percentage point, the $6^{\text {th }}$ harmonic has an amplitude slightly less than $2 \%$. It is possible that this harmonic component, not included in the available HB solutions, is responsible for the deviations of the 3 and 4 modes HB solutions during the upstroke motion. The frequency spectrum of the moment coefficient includes up to the $6^{\text {th }}$ harmonic frequency, whose magnitude is around $5 \%$ the magnitude of the $1^{\text {st }}$ harmonic. It is identified that the $2^{\text {nd }}$ and $3^{r d}$ harmonics greatly affect the moment loop since the magnitude is $46 \%$ and $18 \%$, respectively, the magnitude of the fundamental harmonic.

Dynamic derivatives of the normal force and pitching moment coefficients are summarized in Table 8 for all numerical results. However, the out-of-phase component of the force and moment coefficients measured in different wind tunnel facilities is included to assess the numerical results against experimental data. The experimental data 


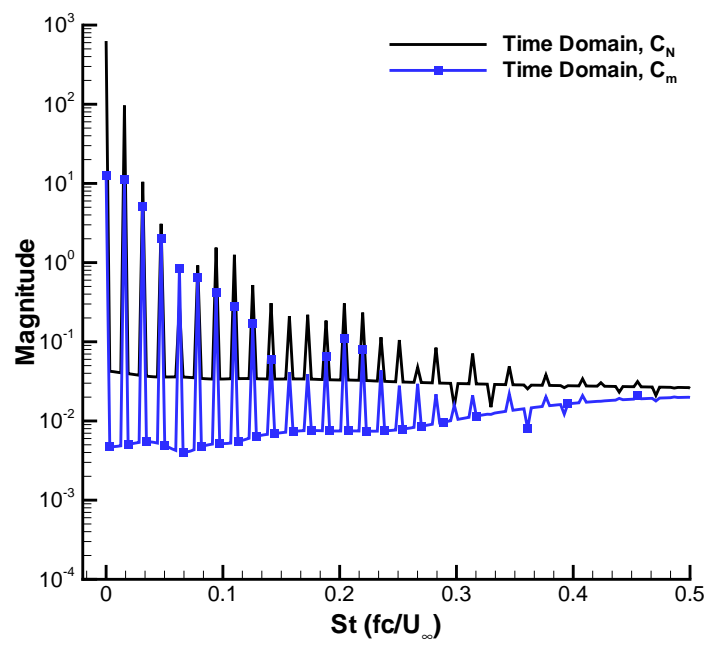

Figure 17. Test Case 2: magnitude of normal force and pitching moment coefficients for the SDM model geometry

for the normal force damping derivative were measured in the continuous, open circuit transonic wind tunnel of the aerodynamic lab at IHU, Iran. ${ }^{28}$ The pitch damping derivatives were measured in the low speed, closed circuit Ankara wind tunnel. ${ }^{27}$ The time-domain damping derivatives agree well with the experimental values. Because of the broad frequency content of the force and moment coefficient, deviations are expected in the predicted values of dynamic derivative for solutions using 1 and 2 modes. One can see that the percentage error in the prediction is reduced as more harmonics are retained in the HB solution. Using as few as 3 modes, a percentage error lower than $10 \%$ with respect to the time-domain values is achieved. The error is further reduced to less than $4 \%$ when 4 modes are included in the HB solution.

\begin{tabular}{lcccc}
\hline & $C_{N_{\alpha}}-k^{2} C_{N_{\dot{q}}}$ & $C_{N_{q}}+C_{N_{\dot{\alpha}}}$ & $C_{m_{\alpha}}-k^{2} C_{m_{\dot{q}}}$ & $C_{m_{q}}+C_{m_{\dot{\alpha}}}$ \\
\hline \hline Experimental & - & 18.7 & - & -5.62 \\
Time Domain: PMB & 3.11 & 15.4 & -0.29 & -5.12 \\
HB - 1 mode & 2.98 & 20.5 & -0.36 & -2.05 \\
HB - 2 modes & 2.98 & 16.6 & -0.35 & -4.51 \\
HB - 3 modes & 3.11 & 14.5 & -0.28 & -5.57 \\
HB - 4 modes & 3.08 & 14.9 & -0.30 & -5.31 \\
\hline
\end{tabular}

Table 8. Test Case 2: dynamic derivatives for the SDM model geometry; experimental data ${ }^{27,28}$ are also included

With strong non-linearities and vortical flows development, a challenging test case was considered to assess the validity and limitations of the HB method. It was demonstrated that the integrated aerodynamic loads contained several modes that were excited by the combination of flow conditions and prescribed oscillatory motion. Nonetheless, the prediction of the aerodynamic loops revealed a good agreement for the solutions using 3 and 4 modes. Similarly, the prediction of the dynamic derivatives using the same number of Fourier modes achieved a deviation of less than $4 \%$ with respect to the reference values.

\section{Test Case 3: Transonic regime}

The acceleration methods and the time-accurate solutions were tested for the prediction of the damping in pitch derivative for the SDM in the transonic regime. The numerical results are compared with experimental data. The flow conditions given in Table 6 feature a Mach number from 0.4 up to 1.1 at a constant value of reduced frequency. The flow field solutions at the steady state conditions at higher Mach numbers contain oblique shock waves forming over the sharp leading and trailing edges. The applied motion causes the motion of the shocks. 
Fig. 18 shows the damping in pitch derivative as a function of the Mach number. The values based on the timeaccurate solutions and the LFD and HB results using 1, 2 and 3 harmonics are included. A comparison is also done against available experimental data from wind tunnel and range data of Winchenbach et al. ${ }^{31}$ An excellent agreement between the time-domain and the HB solutions is achieved throughout the transonic regime. The solution using 3 harmonics exactly reproduces the values from the unsteady calculation. The solution using 2 modes is virtually identical to that using 3 modes. The pitch damping derivatives are slightly overestimated (less negative) for 1 harmonic, however the dip value is in good agreement with other values. The present numerical results agree well with experimental data, clearly showing the sudden increment in the damping near Mach 1. Converged solutions were obtained for the TAU and the LFD methods up to Mach 0.9.

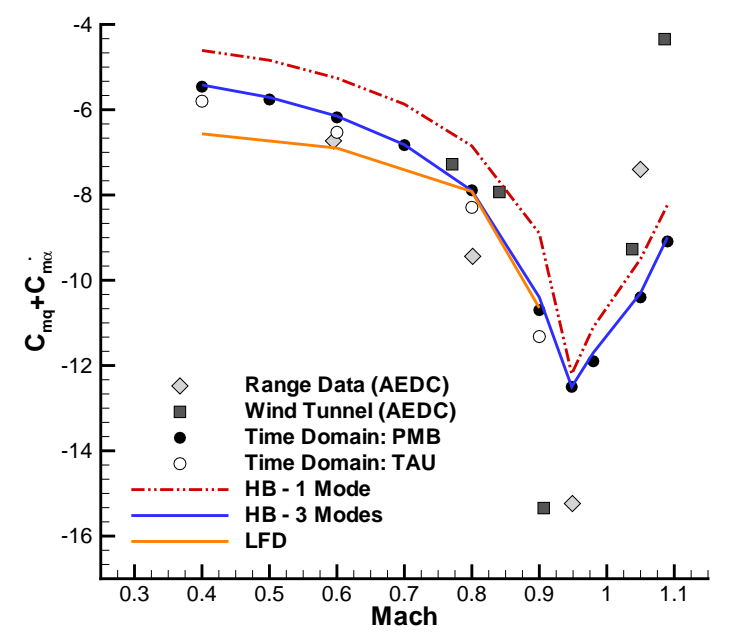

Figure 18. Test Case 3: damping in pitch derivative throughout the transonic regime for the SDM model geometry 


\section{DLR-F12 Wind Tunnel Model}

Within the European Research Project SimSAC, experimental measurements ${ }^{33}$ were obtained on the F12 wind tunnel model from the German Aerospace Center (DLR). Wind tunnel data included static and dynamic force measurements and steady and unsteady pressure distribution measurements. The investigations on the DLR-F12 wind tunnel model were performed in the $3 m \times 3 m$ atmospheric low speed wind tunnel of the German-Dutch Tunnels (DNW-NWB) in Braunschweig a . A benchmark of aerodynamic predictions based on time-domain solutions was reported for the DLR-F12 configuration. ${ }^{34}$

The model consists of wing, body, horizontal and vertical tail. The DLR-F12 wind tunnel model is a 1:40 scale development model of a passenger jet. The CAD model includes a detailed blend at the wing root and an advanced supercritical aerofoil section. The fuselage nose, the tail surfaces and the wing were equipped with transition devices, made from self-adhesive aluminium tape of $0.2 \mathrm{~mm}$ thickness. Wind tunnel wall corrections were applied to static force and pressure distribution measurements. Wall corrections for the dynamic tests were not available in DNWNWB. Static tests were performed in the closed test section, while dynamic tests were run in the slotted test section with a slot ratio of $6 \%$.

The surface grids for the PMB and HB solvers are shown in Fig. 19. The structured grid consists in total of 299320 grid points and the number of surface blocks is 120. A sting is also modeled. Comparison against wind tunnel data is made for the PMB and HB solvers only.

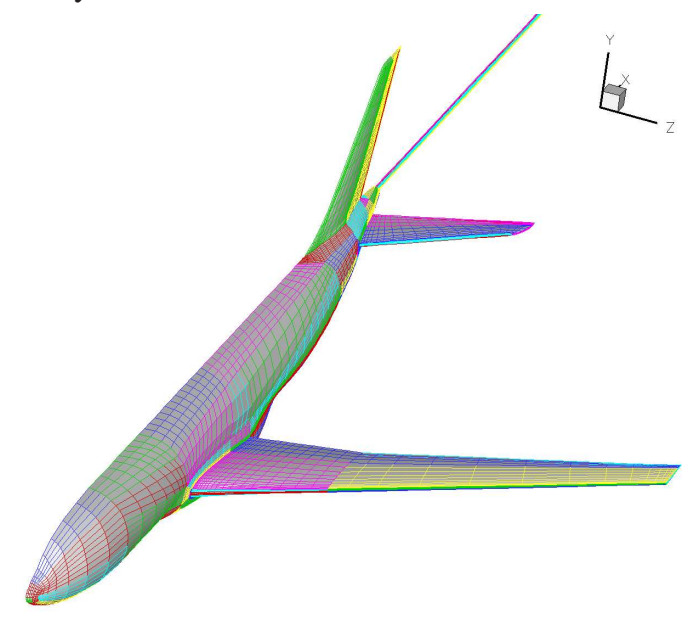

(a) Block structured grid for the PMB and HB solvers

Figure 19. View of the surface grids for the DLR-F12 wind tunnel model

The wind tunnel model dimensions are summarized in Table 9. The reference length for calculating the static and dynamic coefficients of the roll, pitch and yaw moments is the wing reference chord, $c$. The reduced frequency of the oscillatory pitch motion was defined with respect to the mean aerodynamic chord, $c$. The numerical solutions were computed for half model configuration by use of symmetry conditions. In the experimental tests, the reference point for the moments and the rotation centre are coincident. In a coordinate system where $\mathrm{x}$-axis extends from the fuselage nose to the tail, $y$-axis points toward the right wing, and z-axis directed upward the symmetry plane, the reference point has dimensional coordinates $(1.0400008 \mathrm{~m}, 0.0 \mathrm{~m},-0.030285 \mathrm{~m})$.

\begin{tabular}{ll}
\hline \hline Reference surface, $S$ & $0.44414 \mathrm{~m}^{2}$ \\
Mean aerodynamic chord, $c$ & $0.2526 \mathrm{~m}$ \\
Full wing span, $b$ & $2.018 \mathrm{~m}$ \\
\hline \hline
\end{tabular}

Table 9. Reference values of the DLR-F12 wind tunnel model

The PMB and the HB predictive capabilities are validated for forced oscillations in pitch. Experimental measurements are included for two values of freestream speed and mean angle of attack between $0.0^{\circ}$ up to $6.0^{\circ}$. Several

ahttp://www.dnw.aero/home.aspx 
values of frequency of motion were also considered. Numerical results were performed for several permutations of motion parameters, as summarized in Table 10. For the HB method, up to 3 harmonics were retained in the analyses.

\begin{tabular}{llll}
\hline \hline & Test Case 1 & Test Case 2 & Test Case 3 \\
\hline Freestream speed, $U$ & $56 \mathrm{~m} / \mathrm{s}$ & $70 \mathrm{~m} / \mathrm{s}$ & $70 \mathrm{~m} / \mathrm{s}$ \\
Mean incidence, $\alpha_{0}$ & $0.0^{\circ}$ up to $6.0^{\circ}$ & $0.0^{\circ}$ up to $6.0^{\circ}$ & $0.0^{\circ}$ \\
Pitch amplitude, $\alpha_{A}$ & $4.5^{\circ}$ & $3.0^{\circ}$ & $4.5^{\circ}$ \\
Frequency, $f$ & $2.4 \mathrm{~Hz}$ & $3.0 \mathrm{~Hz}$ & $0.5 \mathrm{~Hz}$ up to $3.0 \mathrm{~Hz}$ \\
\hline \hline
\end{tabular}

Table 10. Description of the DLR-F12 test cases for small amplitude pitch oscillations

Fig. 20 shows the damping derivatives for the force and moment coefficients. A first comment is for the good agreement achieved by the time-domain results for all test cases. The small offset between time-accurate and experimental data is due to wind-tunnel interference effects and the neglect of viscous forces in the numerical modelling. At the higher end of the mean angle of attack range, the 1 mode HB solution overpredicts the magnitude of the damping values. The energy of the aerodynamics modes extends beyond the excitation frequency predescribed by the model motion because of moderate non-linear features that were observed in static analyses as well. The energy redistribution at higher frequencies is predicted well retaining 2 Fourier modes. Most importantly, the predictive capabilities of the 2 mode HB solution is equivalent to time-accurate solutions. The increasing deviations in damping values for the 1 and 2 mode HB solutions at the lower end of the frequency range are expected. It is well established that the higher the frequency of motion the higher the hysteresis in the aerodynamic loops because of the time lag in the flowfield adapting to changes in geometry. For slow motions, the loops reduce to a line overlapping the static curve with a small out-of-phase component. These considerations posed the important question to synchronize the balance and position data to less than $100 \mu$ s during experimental measurements. ${ }^{33}$

\section{Conclusions}

Capabilities of the Harmonic Balance method, in the framework of an implicit and explicit multigrid implementation, and the Linear Frequency Domain method are demonstrated for several AGARD CT cases for the pitching NACA 0012 airfoil. For the formation of moving shock waves, the energy of aerodynamic modes redistribute at higher frequencies than the predescribed frequency of motion. In general, the HB method is able to predict the dynamic response data very accurate. In case of the LFD it can be seen that the prediction is less accurate whenever amplitudes increases and compressibility effects apart from the initial steady state solution appear. In terms of pressure distribution, convergence to time-accurate results is also assessed for increasing number of Fourier modes in the HB solution. Differences for the zeroth harmonic unsteady pressure distribution and the steady state solution with the presence of strong dynamic non-linearities demonstrates that the assumptions of the LFD method may experience a loss in accuracy in predicting dynamic response data and pressure distributions. Numerical experiments for these cases confirm that the HB and the LFD methods are an order of magnitude more efficient than time-accurate methods.

The motivation for selecting three-dimensional problems for the flow around a generic fighter configuration and a civil jet aircraft is to highlight the presumed advantages of the HB methodology over the conventional time-domain approach when a fine time discretization is required. The demonstration of the non-linear HB method is stressed in conditions featuring onset of vortical structures and up to the high transonic range for the SDM. A benchmark against experimental data for the DLR-F12 is also considered for benign flows. Choosing not to resolve all the temporal modes in the solution, the HB method achieves a computational efficiency of three orders of magnitude compared to dual-time stepping methods. When the Fourier base includes up to 3 harmonics, the speed up is still as high as one order of magnitude. For the DLR-F12, the reduced order model shows the practical application of complementing extensive wind tunnel campaign.

\section{Acknowledgements}

The work at Liverpool was supported by the SimSAC project under funding from the Sixth Framework programme of the European Union, and the Engineering and Physical Sciences Research Council and the Ministry of Defence under EP/D504473/1, and the ECERTA project under funding from the Sixth Framework MArie Curie Excellence 


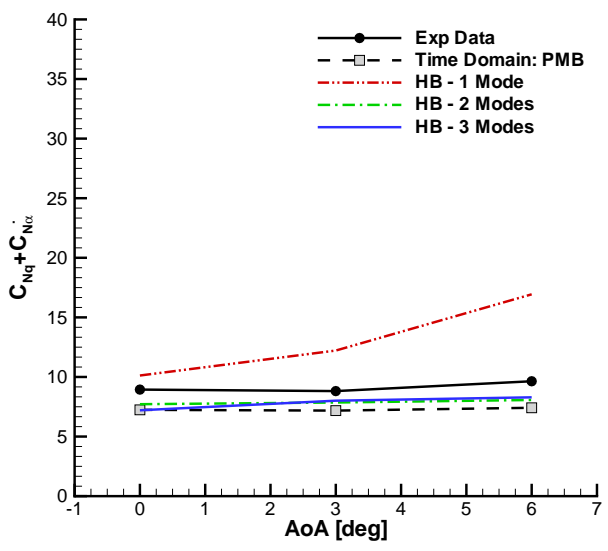

(a) Normal force coefficient damping, Test Case 1

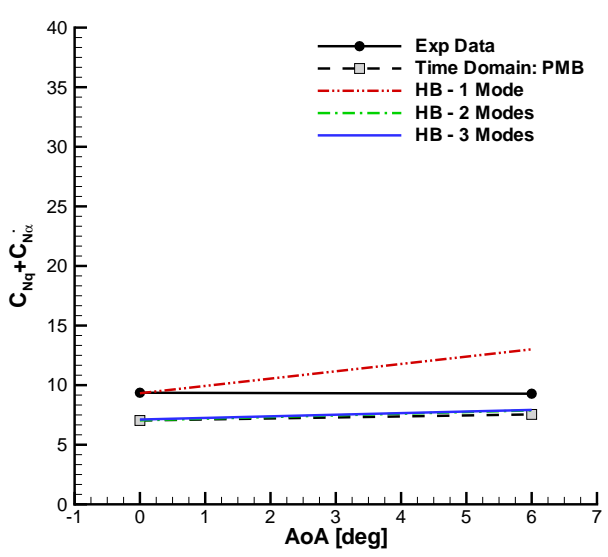

(c) Normal force coefficient damping, Test Case 2

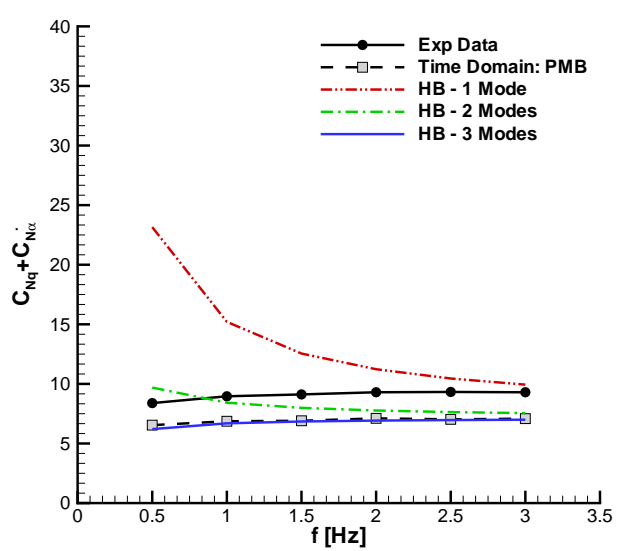

(e) Normal force coefficient damping, Test Case 3

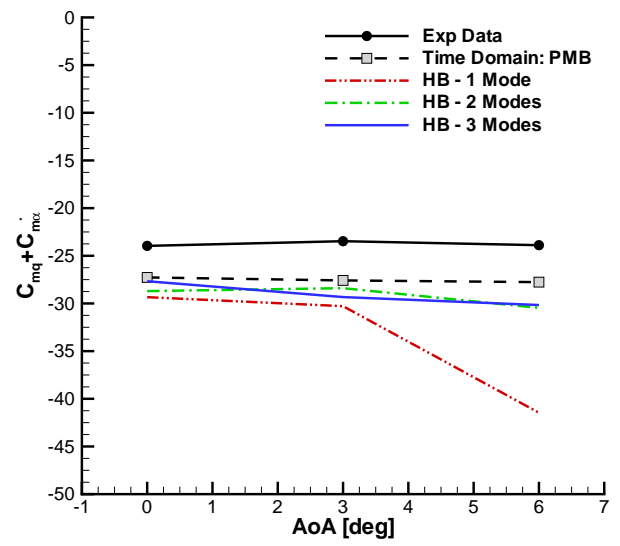

(b) Pitching moment coefficient damping, Test Case 1

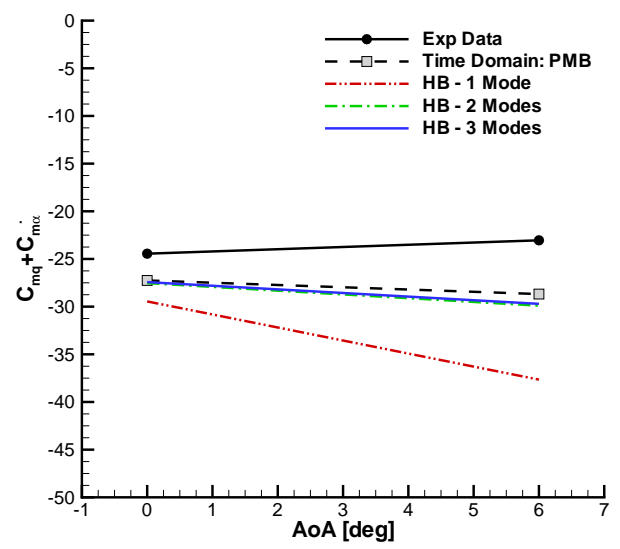

(d) Pitching moment coefficient damping, Test Case 2

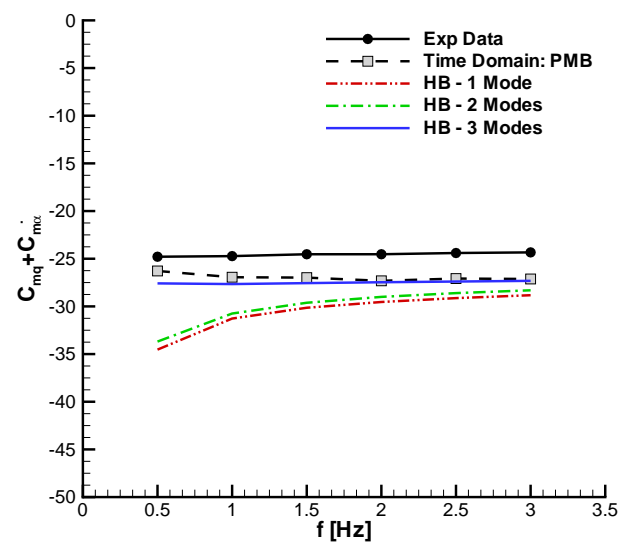

(f) Pitching moment coefficient damping, Test Case 3

Figure 20. Dynamic derivatives for DLR-F12 wind tunnel geometry undergoing forced oscillations in pitch for Test Case $\mathbf{1}(U=56 \mathrm{~m} / \mathrm{s}$, $\alpha_{A}=4.5^{\circ}$ and $\left.f=2.4 \mathrm{~Hz}\right)$, Test Case $2\left(U=70 \mathrm{~m} / \mathrm{s}, \alpha_{A}=3.0^{\circ}\right.$ and $\left.f=3.0 \mathrm{~Hz}\right)$ and Test Case $3\left(U=70 \mathrm{~m} / \mathrm{s}, \alpha_{0}=0.0^{\circ}\right.$ and $\left.\alpha_{A}=4.5^{\circ}\right)$ 
Team. The work at Glasgow was supported by the Engineering and Physical Sciences Research Council under grant $\mathrm{EP} / \mathrm{F} 038542 / 1$.

\section{References}

${ }^{1}$ Bryan, G. H., "Stability in Aviation,” MacMillan, London, 1911.

${ }^{2}$ Bandu, N. P., Performance, Stability, Dynamics, and Control of Airplanes, Second Edition, AIAA Education Series, Blacksburg, Virginia, January 2004.

${ }^{3}$ Park, M. A. and Green, L. L., "Steady-State Computation of Constant Rotational Rate Dynamic Stability Derivatives," 18th AIAA Applied Aerodynamics Conference, AIAA-2000-4321, August 2000.

${ }^{4}$ Williams, J. E. and Vukelich, S. R., "The USAF Stability and Control Digital DATCOM," McDonnell Douglas Astona UTICS Company, St Louis Division, St Louis, Missouri, 63166, 1979, AFFDL-TR-79-3032.

${ }^{5}$ Badcock, K. J., Richards, B. E., and Woodgate, M. A., "Elements of Computational Fluid Dynamics on Block Structured Grids using Implicit Solvers," Progress in Aerospace Sciences, Vol. 36, 2000, pp. 351-392.

${ }^{6}$ Osher, S. and Chakravarthy, S., "Upwind Schemes and Boundary Conditions with Applications to Euler Equations in General Geometries," Journal of Computational Physics, Vol. 50, No. 3, 1983, pp. 447-481.

${ }^{7}$ Van Leer, B., "Towards the Ultimate Conservative Difference Scheme II: Monotonicity and Conservation Combined in a Second Order Scheme," Journal of Computational Physics, Vol. 14, No. 4, 1974, pp. 361-374.

${ }^{8}$ Jameson, A., "Time Dependent Calculations Using Multigrid, with Applications to unsteady Flows Past Airfoils and Wings," AIAA Paper 91-1596, 1991.

${ }^{9}$ Gerhold, T., Galle, M., Friedrich, O., and Evans, J., "Calculation of Complex Three-Dimensional Configurations employing the DLR TAUCode," AIAA Paper 97-0167, 1997.

${ }^{10}$ Schwamborn, D., Gerhold, T., and Heinrich, R., "The DLR TAU-Code: Recent Applications in Research and Industry." ECCOMAS CFD 2006, TU Delft, The Netherlands, 2006.

${ }^{11}$ Dwight, R., Brezillon, J., and Vollmer, D., "Efficient Algorithms for Solution of the Adjoint Compressible Navier-Stokes Equations with Applications," Proceedings of the ONERA-DLR Aerospace Symposium (ODAS), Toulouse, 2006.

${ }^{12}$ Campobasso, M., Bonfiglioli, A., and Baba-Ahmadi, M., "Development of Efficient and Accurate CFD Technologies for Wind Turbine Unsteady Aerodynamics," Proceedings of the Conference on Modeling Fluid Flow, edited by J. Vad, Vol. 2 of 14th Event of International Conference Series on Fluid Flow Technologies held in Budapest, Department of Fluid Mechanics, Budapest University of Technology and Economics, September 2009, pp. 879-886.

${ }^{13}$ Bonfiglioli, A., Campobasso, M., and Carpentieri, B., "Parallel Unstructured Three-dimensional Turbulent Flow Analyses Using Efficiently Preconditioned Newton-Krylov Solver," AIAA paper 2009-4137, June 2009, 19th AIAA Computational Fluid Dynamics Conference, San Antonio, Texas.

${ }^{14}$ Campobasso, M., Baba-Ahmadi, M., and McLelland, G., "Ad-hoc Boundary Conditions for CFD Analyses of Turbomachinery Problems with Strong Radial Flow Gradients at Farfield Boundaries," ASME paper GT2010-22176, to appear in the ASME Journal of Turbomachinery, June 2010, ASME Turbo Expo 2010 Gas Turbine Technical Congress and Exposition, Glasgow, United Kingdom.

${ }^{15}$ Hall, K. Thomas, J. and Clark, W., "Computation of Unsteady Nonlinear Flows in Cascades Using a Harmonic Balance Technique," AIAA Journal, Vol. 40, No. 5, May, 2002, pp. 879-886.

${ }^{16}$ Thomas, J. P., Dowell, E. H., Hall, K. C., and Denegri, C. M., "Further Investigation of Modeling Limit Cycle Oscillation Behaviour of the F-16 Fighter Using a Harmonic Balance Approach," AIAA Paper 2005-1917, Austin, TX, April 2005, 46th AIAA/ASME/ASCE/AHS/ASC Structures, Structural Dynamics and Materials (SDM) conference.

${ }^{17}$ Woodgate, M. and Badcock, K., "Implicit Harmonic Balance Solver for Forced Motion Transonic Flow," AIAA Journal, Vol. 47, No. 4, 2008, pp. 893-901.

${ }^{18}$ Pechloff, A. and Laschka, B., "Small Disturbance Navier-Stokes Method: Efficient Tool for Predicting Unsteady Air Loads." Journal of Aircraft, Vol. 43, No. 1, 2006, pp. 17-29.

${ }^{19}$ Widhalm, M., Dwight, R. P., Thormann, R., and Hübner, A. R., "Efficient Computation of Dynamic Stability Data with a Linearized Frequency Domain Solver." ECCOMAS CFD 2010, Lisbon, Portugal, 2006.

${ }^{20}$ Da Ronch, A., Vallespin, D., Ghoreyshi, M., and Badcock, K. J., "Computation of Dynamic Derivatives Using CFD," 28th AIAA Applied Aerodynamics Conference, AIAA-2010-4817, Chicago, IL, 2010.

${ }^{21}$ Landon, R. H., "NACA 0012. Oscillating and Transient Pitching," Compendium of Unsteady Aerodynamic Measurements, Data Set 3, AGARD-R-702, August 1982.

22 "Verification and Valiation Data for Computational Unsteady Aerodynamics," Reserch and Technology Organization, RTO-TR-26, October, 2000.

${ }^{23}$ Tijdeman, H. and Seebass, R., "Transonic Flow Past Oscillating Airfoils," Annual Review of Fluid Mechanics, Vol. 12, 1980, pp. 181-222.

${ }^{24}$ Dufour, G., Sicot, F., and Puigt, G., "Contrasting the Harmonic Balance and Linearized Methods for Oscillating-Flap Simulations," AIAA Journal, Vol. 48, No. 4, April 2010.

${ }^{25}$ Beyers, M. E., "Subsonic Roll Oscillation Experiments on the Standard Dynamics Model," AIAA Atmospheric Flight Mechanics Conference, AIAA-83-2134, 1983

${ }^{26}$ Jermey, C. and Schiff, L., "Wind Tunnel Investigation of the Aerodynamic Characterestics of the Standard Dynamic Model in Coning Motion at Mach 0.6," 12th Atmospheric Flight Mechanics Conference, AIAA-85-1828, 1985.

${ }^{27}$ Alemdaroglu, N., Iyigun, I., Altun, M., Uysal, H., Quagliotti, F., and Guglieri, G., "Determination of Dynamic Stability Derivatives Using Forced Oscillation Technique," 40th Aerospace Science Meeting and Exhibition, AIAA-2002-0528, 2002.

${ }^{28}$ Davari, A. R. and Soltani, M. R., "Effects of Plunging Motion on Unsteady Aerodynamic Behaviour of an Aircraft Model in Compressible Flow," Iranian Journal of Science and Technology, Transaction B Enginneering, Vol. 31, No. B1, 2007, pp. 49-63.

${ }^{29}$ Huang, X. Z., "Wing and Fin Buffet on The Standard Dynamic Model," NATO RTO Report Number, RTO-TR-26, pp. 361-381. 
${ }^{30}$ Ghoreyshi, M., Badcock, K. J., Da Ronch, A., Swift, A., Marques, S., and Ames, N., "Framework for Establishing the Limits of Tabular Aerodynamic Models for Flight Dynamics," AIAA Atmospheric Flight Mechanics Conference, AIAA-2009-6273, 2009.

${ }^{31}$ Winchenbach, G. L., Uselton, B. L., Hathaway, W. H., and Chelekis, R. M., "Comparison of Free-Flight and Wind Tunnel Data for a Generic Fighter Configuration,” AIAA Paper 82-1365, 1982.

${ }^{32}$ Murman, S. M., "A Reduced-Frequency Approach for Calculating Dynamic Derivatives," 43rd AIAA Aerospace Sciences Meeting and Exhibit - Meeting Papers, AIAA-2005-0840, 2005, pp. 3211-3227.

${ }^{33}$ Hübner, A. R., "Final Summary of Wind Tunnel Test (Steady/Unsteady Force and Pressure Measurements of the DLR-F12 Model in the DNW-NWB within the European Research Project SimSAC)," SimSAC-Task 4.1-1, Delivery Number D 4.1-1, 20 April 2008.

${ }^{34}$ Mialon, B., Ben Khelil, S., Huebner, A., Jouhaud, J.-C., Roge, G., Hitzel, S., Badcock, K., Eliasson, P., Khabrov, A., and Lahuta, M., "European Benchmark on Numerical Prediction of Stability and Control Derivatives," 27th AIAA Applied Aerodynamics Conference, AIAA-20094116, San Antonio, TX, 2009. 